\title{
A Comparison of Accuracy and Computational Efficiency of Three Pseudospectral Methods
}

\author{
Geoffrey T. Huntington* \\ David Benson ${ }^{\dagger}$ \\ Charles Stark Draper Laboratory \\ 555 Technology Square \\ Cambridge, MA 02139 \\ Anil V. Rao ${ }^{\ddagger}$ \\ University of Florida \\ Gainesville, FL 32611-6250
}

\begin{abstract}
A comparison is made between three pseudospectral methods used to numerically solve optimal control problems. In particular, the accuracy of the state, control, and costate obtained using the Legendre, Radau, and Gauss pseudospectral methods is compared. Three examples with different degrees of complexity are used to identify key differences between the three methods. The results of this study indicate that the Radau and Gauss methods are very similar in accuracy, while both significantly outperform the Legendre method with respect to costate accuracy. Furthermore, it is found that the computational efficiency of the three methods is comparable. Based on these results and a detailed analysis of the mathematics of each method, a rationale is created to determine when each method should be implemented to solve optimal control problems.
\end{abstract}

\section{Introduction}

Numerical methods for solving optimal control problems fall into two general categories: indirect methods and direct methods. In an indirect method, the first-order optimality conditions are derived using the calculus of variations ${ }^{1}$ and Pontryagin's minimum principle. ${ }^{2}$ These optimality conditions lead to a Hamiltonian boundary-value problem (HBVP) which is then solved numerically to determine extremal trajectories. In a direct method, the original optimal control problem is transcribed to a nonlinear programming problem (NLP) which is solved numerically using a well-established optimization method. In recent years, direct methods for solving optimal control problems have become increasingly popular over indirect methods for several reasons. First, when using a direct method it is unnecessary to derive the (generally quite complex) first-order optimality conditions (i.e. HBVP). Second, even in the case where deriving the HBVP is straightforward, solving the HBVP can be extremely difficult due to the high sensitivity of the Hamiltonian dynamics to unknown boundary conditions.

\footnotetext{
* Draper Laboratory Fellow, Ph.D. Candidate, Dept. of Aeronautics and Astronautics, MIT. E-mail: ghuntington@draper.com.

${ }^{\dagger}$ Draper Laboratory Fellow. E-mail: bensonda@alum.mit.edu

${ }_{\ddagger}^{\ddagger}$ Assistant Professor, Department of Mechanical and Aerospace Engineering, University of Florida, P.O. Box 116250, Gainesville, FL, 32611. E-mail: anilvrao@ufl.edu. Corresponding Author.
} 
Contrariwise, direct methods require much less mathematical derivation and the resulting NLP can be solved with much greater ease as compared to the HBVP. For these reasons, direct methods are often the preferred approach for numerically solving complex optimal control problems. ${ }^{3}$

The category of direct methods is quite broad and encompasses some very different techniques. ${ }^{4}$ In particular, the choice of what quantities to discretize and how to approximate the continuoustime dynamics varies widely amongst the different direct methods. Two of the more common direct methods are control parameterization and state and control parameterization. ${ }^{5}$ In a control parameterization method, the control alone is approximated and the differential equations are solved via numerical integration. Examples of control parameterization include shooting methods and multiple shooting methods. In state and control parameterization methods, the state is discretized in the NLP as well as the control, and the continuous-time differential equations are converted into algebraic constraints. These constraints are then imposed in the NLP formulation, which avoid the sensitivity issues of direct shooting methods at the expense of a larger NLP.

In recent years, considerable attention has been focused on a class of state and control parameterization methods called pseudospectral ${ }^{6-8}$ or orthogonal collocation ${ }^{9,10}$ methods. In a pseudospectral method, a finite basis of global interpolating polynomials is used to approximate the state and control at a set of discretization points. The time derivative of the state in the dynamic equations is approximated by the derivative of the interpolating polynomial and is then constrained to be equal to the vector field of the dynamic equations at a set of collocation points. While any set of unique collocation points can be chosen, generally speaking an orthogonal collocation is chosen, i.e. the collocation points are chosen to be the roots of an orthogonal polynomial (or linear combinations of such polynomials and their derivatives). Because pseudospectral methods are generally implemented via orthogonal collocation, the terms pseudospectral and orthogonal collocation are essentially interchangeable (thus researchers in one field use the term pseudospectral $^{8}$ while others use the term orthogonal collocation $^{9}$ ). One advantage to pseudospectral methods is that for smooth problems, pseudospectral methods typically have faster convergence rates than other methods, exhibiting so called "spectral accuracy". ${ }^{11}$ For non-smooth problems or problems where modeling changes are desired, the optimal control problem can be divided into phases and orthogonal collocation can be applied globally within each phase. A vast amount of work has been done on using pseudospectral methods to solve non-smooth optimal control problems (see Refs. 12-17).

Pseudospectral methods in optimal control arose from spectral methods which were traditionally used to solve fluid dynamics problems. ${ }^{7,8}$ Meanwhile, seminal work in orthogonal collocation methods in optimal control date back to 1979 with the work of Ref. 18 and some of the first work using orthogonal collocation methods in engineering can be found in the chemical engineering literature. ${ }^{17}$ More recent work in chemical and aerospace engineering have used collocation at the Legendre-GaussRadau (LGR) points. ${ }^{19-21}$ Because the LGR collocation technique has many similarities to other pseudospectral methods, in this paper we adopt the term Radau pseudospectral method (RPM) for LGR collocation. Within the aerospace engineering community, several well-known pseudospectral methods have been developed for solving optimal control problems such as the Chebyshev pseudospectral method (CPM), ${ }^{22,23}$ the Legendre pseudospectral method (LPM), ${ }^{6}$ and the Gauss pseudospectral method (GPM). ${ }^{24}$ The CPM uses Chebyshev polynomials to approximate the state and control, and performs orthogonal collocation at the Chebyshev-Gauss-Lobatto (CGL) points. An enhancement to the Chebyshev pseudospectral method that uses a Clenshaw-Curtis quadrature was developed in Ref. 30. The LPM uses Lagrange polynomials for the approximations, and Legendre-Gauss-Lobatto (LGL) points for the orthogonal collocation. A costate estimation procedure for the Legendre pseudospectral method was developed by in Ref. 25 and recently updated in Ref. 26. Recent work by Williams shows several variants of the standard LPM. The Jacobi pseudospectral method ${ }^{27}$ is a more general pseudospectral approach that uses Jacobi polynomials to find the collocation points, of which Legendre polynomials 
are a subset. Another variant, called the Hermite-LGL method, ${ }^{28}$ uses piecewise cubic polynomials rather than Lagrange polynomials, and collocates at a subset of the LGL points. These new methods have not yet become mainstream optimization techniques because of their novelty, but the new software tool DIRECT ${ }^{29}$ could expedite this process. Lastly, in the GPM, the state is approximated using a basis of Lagrange polynomials similar to the LPM, and the optimal control problem is orthogonally collocated at the interior Legendre-Gauss (LG) points.

Two key features of these pseudospectral methods is the choice of discretization points and collocation points. Discretization points (or nodes) are the points used to discretize the state and control that are fed into the nonlinear program. Collocation points are the points used to collocate the differential equations to ensure the dynamics have been met. As we will see, these two sets of points drastically affect the accuracy of the methods. Among the pseudospectral methods previously mentioned, a unique quality exists for the Gauss pseudospectral method. The specific choice of collocation and discretization points in the GPM results in an equivalence between the Karush-Kuhn-Tucker (KKT) conditions of the NLP and the discretized form of the first-order optimality conditions at the LG points. In other words, the solution of the NLP (direct approach) also satisfies the same first-order conditions if one were using an indirect approach. ${ }^{24}$ A diagram of this equivalence for the GPM is shown in Fig. 1. Although an exact equivalence has not been proven for the other methods, much research has been done on both the LPM and the RPM that discusses the (rapid) convergence rates of the NLP solution to the solution of the continuous-time optimality conditions. ${ }^{19,26,31}$ Moreover, for both the LPM and the RPM a relationship between the KKT conditions and the discretized first-order necessary conditions leads to method for costate approximation based on the KKT multipliers of the NLP. These costate approximation procedures have been documented for the RPM in Ref. 19, for the LPM in Ref. 25,26, and for the GPM in Ref. 24. The accuracy of these procedures, as well as the state and control approximations themselves, are compared in this paper for the GPM, LPM and RPM.

Numerical comparisons between methods are often difficult to perform fairly because many direct methods are integrated with a specific NLP solver, thereby making it difficult to distinguish between differences between the methods and differences due to the NLP solvers. This is perhaps why there are relatively few papers that actually compare the performance of several direct methods. In fact, even survey papers, such as that found in Ref. 3, stop short of providing comparisons between various methods. However, some research has been done that compares direct methods. Ref. 32 is primarily focused on presenting enhanced mesh refinement and scaling strategies, but contains a brief comparison of the accuracy of direct implicit integration schemes (like Hermite-Simpson integration) and pseudospectral methods. Williams created the software tool DIRECT in order to more effectively compare various trajectory optimization algorithms. The results of a thorough comparison involving 21 test problems of several different direct methods is presented in Ref. 29. Ref. 33 presents a comparison between several pseudospectral approaches, but uses unorthodox variants of pseudospectral methods that are inconsistent with a large majority of the literature.

In this paper a comparison is made between the Legendre, Radau, and Gauss pseudospectral methods. In order to provide a fair comparison, in this study the NLP solver SNOPT ${ }^{39}$ is used for each of the discretizations. Furthermore, all three methods are implemented using the same version of MATLAB ${ }^{\circledR}$ and the initial guesses provided for all examples are identical. Using this equivalent setup for all three methods, the goal of the study is to assess the similarities and differences in the accuracy and and computational performance between the three methods. Three examples are used to make the comparison. The first two examples are designed to be sufficiently simple so that the key features of each method can be identified. The third example, a commonly used problem in aerospace engineering, is designed to provide an assessment as to how the three methods compare on a more realistic problem. A great deal of the emphasis of this study is to understand when one method may perform better than another method and to identify why such an improved performance is attained 
in such circumstances.

The results of this study indicate that the Radau and Gauss pseudospectral methods are highly comparable in numerical accuracy, computational efficiency, and ease of use. The largest discrepancy in accuracy between these two methods corresponds to the costate at the final time, where the natural equivalence in the GPM leads to a more accurate final costate. This study also shows that, even on simple problems, both the Radau and Gauss methods produce higher accuracy solutions as compared to the Legendre method. Specifically, the costate estimates obtained using the Radau and Gauss methods are superior to the costate estimate obtained using the established method of Ref. 25. The authors recognize that improvements to these methods are still occurring, therefore a second computation is performed using a recent advancement of the Legendre pseudospectral method. The augmented set of KKT conditions ${ }^{26}$ is solved using the Legendre method with the covector mapping theorem, and the resulting costate is compared with the Radau and the Gauss methods. While it is found that this new costate is significantly more accurate than the original costate obtained using the method of Ref. 25, it still does not provide costate accuracies comparable to the GPM and RPM for a given number of discretization points. Furthermore, it is argued that such an approach is highly intractable because it requires formulating a primal-dual problem involving the transversality conditions of the HBVP and the (generally quite complex) KKT conditions of the NLP, thus defeating the purpose of costate estimation using the solution from a direct method.

The increased accuracy in the Radau and Gauss costates arises from the fact that no collocation occurs at least one of the endpoints in these two methods. As a result, the conflict ${ }^{26}$ that arises in the Legendre method between the transversality conditions and the discretized dynamics and quadrature approximation is eliminated or mitigated. The results of this study suggest that the Gauss method produces the most accurate costate estimates, followed closely by the Radau method. Depending on the costate approximation procedure chosen for the Legendre method, it produces either a significantly less accurate costate, or requires relatively much more computational expense than either the Radau or Gauss approach.

\section{Continuous Mayer Problem}

Without loss of generality, consider the following optimal control problem in Mayer form. Determine the state, $\mathbf{x}(\tau) \in \mathbb{R}^{n}$, and control, $\mathbf{u}(\tau) \in \mathbb{R}^{m}$, that minimize the cost functional

$$
J=\Phi\left(\mathbf{x}\left(\tau_{f}\right)\right)
$$

subject to the constraints

$$
\begin{gathered}
\frac{d \mathbf{x}}{d \tau}=\frac{t_{f}-t_{0}}{2} \mathbf{f}(\mathbf{x}(\tau), \mathbf{u}(\tau)) \in \mathbb{R}^{n} \\
\boldsymbol{\phi}\left(\mathbf{x}\left(\tau_{0}\right), \mathbf{x}\left(\tau_{f}\right)\right)=\mathbf{0} \in \mathbb{R}^{q}
\end{gathered}
$$

The optimal control problem of Eqs. (1)-(3) will be referred to as the continuous Mayer problem. It is noted that, in order to simplify the computational expense and provide a clear comparison, we consider optimal control problems with fixed terminal times and without algebraic path constraints. Furthermore, it is noted that the time interval $\tau \in[-1,1]$ can be transformed to the the time interval $t \in\left[t_{0}, t_{f}\right]$ via the affine transformation

$$
t=\frac{t_{f}-t_{0}}{2} \tau+\frac{t_{f}+t_{0}}{2}
$$




\section{First-Order Necessary Conditions of Continuous Mayer Problem}

The indirect approach to solving the continuous Mayer problem of Eqs. (1)-(3) in Section 2 is to apply the calculus of variations and Pontryagin's Minimum Principle ${ }^{2}$ to obtain first-order necessary conditions for optimality. ${ }^{1}$ These variational conditions are typically derived using the Hamiltonian, $\mathcal{H}$, defined as

$$
\mathcal{H}(\mathbf{x}, \boldsymbol{\lambda}, \mathbf{u})=\boldsymbol{\lambda}^{T} \mathbf{f}(\mathbf{x}, \mathbf{u})
$$

where $\boldsymbol{\lambda}(\tau) \in \mathbb{R}^{n}$ is the costate. Note that, for brevity, the explicit dependence of the state, control, and costate on $\tau$ has been dropped. Assuming that the optimal control lies interior to the feasible control set, the continuous-time first-order optimality conditions are

$$
\begin{aligned}
& \frac{d \mathbf{x}}{d \tau}=\frac{t_{f}-t_{0}}{2} \mathbf{f}(\mathbf{x}, \mathbf{u})=\frac{t_{f}-t_{0}}{2} \frac{\partial \mathcal{H}}{\partial \boldsymbol{\lambda}} \\
& \frac{d \boldsymbol{\lambda}}{d \tau}=-\frac{t_{f}-t_{0}}{2} \boldsymbol{\lambda}^{T} \frac{\partial \mathbf{f}}{\partial \mathbf{x}}=-\frac{t_{f}-t_{0}}{2} \frac{\partial \mathcal{H}}{\partial \mathbf{x}} \\
& \mathbf{0}=\quad \boldsymbol{\lambda}^{T} \frac{\partial \mathbf{f}}{\partial \mathbf{u}} \quad=\frac{\partial \mathcal{H}}{\partial \mathbf{u}} \\
& \boldsymbol{\phi}\left(\mathbf{x}\left(\tau_{0}\right), \mathbf{x}\left(\tau_{f}\right)\right)=\mathbf{0} \\
& \boldsymbol{\lambda}\left(\boldsymbol{\tau}_{0}\right)=\boldsymbol{v}^{T} \frac{\partial \boldsymbol{\phi}}{\partial \mathbf{x}\left(\tau_{0}\right)}, \boldsymbol{\lambda}\left(\boldsymbol{\tau}_{f}\right)=\frac{\partial \Phi}{\partial \mathbf{x}\left(\boldsymbol{\tau}_{f}\right)}-\boldsymbol{v}^{T} \frac{\partial \boldsymbol{\phi}}{\partial \mathbf{x}\left(\boldsymbol{\tau}_{f}\right)}
\end{aligned}
$$

where $\boldsymbol{v} \in \mathbb{R}^{q}$ is Lagrange multiplier associated with the boundary condition $\boldsymbol{\phi}$. The optimality conditions of Eq. (6) define a Hamiltonian boundary value problem (HBVP).

\section{Pseudospectral Approximation Methods}

Before proceeding to the comparison, it is important to understand the mathematics associated with each pseudospectral method. This section provides an overview of the mathematics implemented in the different methods and highlights the key differences.

\subsection{Global Polynomial Approximations}

All three pseudospectral methods compared in this paper (i.e. Legendre, Radau, and Gauss) employ global polynomial approximations using Lagrange interpolating polynomials as the basis functions. These polynomials are defined using a set of $N$ support points $\tau_{1}, \ldots, \tau_{N}$ on the time interval $\tau \in$ $[-1,1]$. The state, control, and costate of the optimal control problem are all approximated as ${ }^{35}$

$$
y(\tau) \approx Y(\tau)=\sum_{i=1}^{N} \mathcal{L}_{i}(\boldsymbol{\tau}) Y\left(\boldsymbol{\tau}_{i}\right), \quad \text { where } \quad \mathcal{L}_{i}(\boldsymbol{\tau})=\prod_{j=1, j \neq i}^{N} \frac{\boldsymbol{\tau}-\boldsymbol{\tau}_{j}}{\boldsymbol{\tau}_{i}-\boldsymbol{\tau}_{j}}
$$

where $\mathcal{L}_{i}(\tau),(i=1, \ldots, N)$ is the set of Lagrange interpolating polynomials corresponding to the chosen support points and $Y$ represents either the state, control, or costate. It can be shown that

$$
\mathcal{L}_{i}\left(\tau_{j}\right)= \begin{cases}1, & i=j \\ 0 & , \quad i \neq j\end{cases}
$$

Eq. (8) leads to the property that $y\left(\tau_{j}\right)=Y\left(\tau_{j}\right),(j=1, \ldots, N)$, i.e. the function approximation is equal to the true function at the $\mathrm{N}$ points. It is important to keep in mind that the support points 
are different for each of the three pseudospectral methods. Furthermore, within a given method, the number of points used in Eq. (7) can be different for each variable (e.g. the state may be approximated with one basis of Lagrange polynomials, while the control is approximated with a different basis of Lagrange polynomials). See Table 1 for a complete list of the Lagrange polynomial approximations used in this paper.

\subsection{Quadrature Approximation}

In addition to the approximation of the state, control, and costate, each method uses a specific set of support points at which to collocate the dynamics. These support points are chosen to minimize the error in the quadrature approximation ${ }^{36}$ to an integral of a function. The general form for the quadrature approximation in these pseudospectral methods is

$$
\int_{a}^{b} f(\tau) d \tau \approx \sum_{i=1}^{N} w_{i} f\left(\tau_{i}\right)
$$

where $\tau_{1}, \ldots, \tau_{N}$ are the support points of the quadrature on the interval $\tau \in[-1,1]$ and $w_{i},(i=$ $1, \ldots, N)$ are quadrature weights. For an arbitrary set of unique points $\tau_{1}, \ldots, \tau_{N}$, the quadrature approximation is exact for polynomials of degree $N-1$ or less. However, by appropriately choosing the support points, the accuracy of the quadrature can be improved greatly. It is well-known that the quadrature approximation with the highest accuracy for a given number of support points $N$ is the Gauss quadrature. In particular, the Gauss quadrature is exact for all polynomials of degree $2 N-1$ or less. In a Gauss quadrature, the $N$ support points are the Legendre-Gauss (LG) points and are the roots of the $N^{t h}$-degree Legendre polynomial, denoted $P_{N}(t)$. These nonuniform support points are located on the interior of the interval $[-1,1]$, and are clustered towards the boundaries. The corresponding Gauss quadrature weights are then found by the formula

$$
w_{i}=\frac{2}{\left(1-\tau_{i}^{2}\right)\left[\dot{P}_{N}\left(\tau_{i}\right)\right]^{2}}, \quad(i=1, \ldots, N)
$$

where $\dot{P}_{N}$ is the derivative of the $N^{t h}$-degree Legendre polynomial.

As we will see, another approach is to use Legendre-Gauss-Radau (LGR) points, which lie on the interval $\tau \in[-1,1)$. By forcing a support point to lie at the boundary, the degree of freedom is reduced by one, thus making this choice of support points accurate to $2 \mathrm{~N}-2$. The N LGR points are defined as the roots of $P_{N}(\tau)+P_{N-1}(\tau)$, or the summation of the $N$ th and $(N-1)$ th order Legendre polynomials. The corresponding weights for the LGR points are

$$
\begin{aligned}
& w_{1}=\frac{2}{N^{2}}, \\
& w_{i}=\frac{1}{\left(1-\tau_{i}\right)\left[\dot{P}_{N-1}\left(\boldsymbol{\tau}_{i}\right)\right]^{2}}, \quad i=2, \ldots, N
\end{aligned}
$$

The standard set of LGR points includes the initial point but not the final point. This set of LGR points works well for infinite horizon problems. ${ }^{33}$ For finite-horizon problems, the LGR points (and corresponding weights) are often flipped on the interval, ${ }^{21}$ meaning the set includes the final point but not the initial point $(\tau \in(-1,1])$. This set of LGR points can be found from the roots of $P_{N}(\tau)-$ $P_{N-1}(\tau)$.

The third set of support points implemented in this paper are the Legendre-Gauss-Lobatto (LGL) points, which lie on the interval $\tau \in[-1,1]$. In this approach, quadrature points are forced to lie 
at both boundaries, reducing the degree of freedom by an additional one degree, thus making this quadrature scheme accurate to $2 \mathrm{~N}-3$. The N LGL nodes are the roots of $\left(1-\tau^{2}\right) \dot{P}_{N-1}(\tau)$ (where $\dot{P}_{N-1}(\tau)$ is the derivative of the $(N-1)$ th order Legendre polynomial, $\left.P_{N-1}(\tau)\right)$. The corresponding weights for the LGL points are

$$
w_{i}=\frac{2}{N(N-1)} \frac{1}{\left[P_{N-1}\left(\tau_{i}\right)\right]^{2}}, \quad(i=1, \ldots, N)
$$

The mathematics of function approximation and quadrature approximation described in this section will be used in the formulation of all the pseudospectral methods presented in this paper.

\section{Pseudospectral Methods}

The three pseudospectral methods in this comparison approximate the state and control using a finite basis of global polynomials, which are created by first discretizing the problem into $\mathrm{N}$ nodes along the trajectory (described in Section 4). However, the location of these nodes is unique to each method, along with the choice of basis polynomials. These differences are explained in detail in this section. Furthermore, all pseudospectral methods transcribe the dynamic equations into algebraic conditions, which are evaluated at a set of collocation points. These collocation points are also unique to each method and may or may not be the same as the nodes. The locations of nodes and collocation points, as well as the choice of basis functions are key features that distinguish these methods from each other. There are many different types of pseudospectral methods, but this work will be limited to three methods: the Legendre, Radau, and Gauss pseudospectral methods, since these are the three methods with published costate approximation procedures. Lastly, it is noted that the indexing in this section may be slightly different than the indexing contained in the references. This is done in order to create some commonality between the three methods in terms of their indices, and to create NLPs that are equivalent in size. Therefore, in all methods, $\mathrm{N}$ represents the total number of discretization points (nodes) used in the NLP resulting from each pseudospectral discretization. Moreover, in general, the index $j$ will be used to represent the $j$-th node, while the index $k$ will be used to represent the $k$-th collocation point.

\subsection{Legendre Pseudospectral Method (LPM)}

The Legendre pseudospectral method (LPM), like all pseudospectral methods, is based on approximating the state using a finite set of interpolating polynomials. The state is approximated using a basis of $N$ Lagrange interpolating polynomials, $\mathcal{L}_{i}(\tau)(i=1, \ldots, N)$, as defined in Eq. (7).

$$
\mathbf{X}(\boldsymbol{\tau}) \approx \mathbf{X}(\boldsymbol{\tau})=\sum_{i=1}^{N} \mathcal{L}_{i}(\boldsymbol{\tau}) \mathbf{X}\left(\boldsymbol{\tau}_{i}\right)
$$

In the LPM, the location of $N$ nodes are described by the Legendre-Gauss-Lobatto (LGL) points on the interval $\tau \in[-1,1]$. Recall that the endpoints -1 and 1 are included as nodes.

Next, all pseudospectral methods transcribe the dynamic equations by "collocating" the derivative of this state approximation with the vector field, $\mathbf{f}(\mathbf{x}, \mathbf{u})$, at a set of points along the interval. Let us assume there are $K$ points, which we call collocation points. In the LPM, the LGL points are both the nodes and the points used to collocate the dynamics, meaning $N=K$. Mathematically, the derivative of the state approximation at the $k$-th LGL point, $\tau_{k}$, is

$$
\dot{\mathbf{X}}\left(\boldsymbol{\tau}_{k}\right) \approx \dot{\mathbf{X}}\left(\tau_{k}\right)=\sum_{i=1}^{N} \dot{\mathcal{L}}_{i}\left(\boldsymbol{\tau}_{k}\right) \mathbf{X}\left(\boldsymbol{\tau}_{i}\right)=\sum_{i=1}^{N} D_{k i} \mathbf{X}\left(\tau_{k}\right), \quad(k=1, \ldots, K)
$$


where the differentiation matrix, $D \in \mathbb{R}^{K \times N}$, is defined as

$$
D_{k i}= \begin{cases}\frac{P_{N-1}\left(\tau_{k}\right)}{P_{N-1}\left(\tau_{i}\right)} \frac{1}{\tau_{k}-\tau_{i}}, & \text { if } k \neq i \\ -\frac{(N-1) N}{4}, & \text { if } k=i=1 \\ \frac{(N-1) N}{4}, & \text { if } k=i=N \\ 0, & \text { otherwise }\end{cases}
$$

The continuous dynamics of Eq. (2) are then transcribed into the following set of $N$ algebraic equations via collocation:

$$
\sum_{i=1}^{N} D_{k i} \mathbf{X}\left(\tau_{i}\right)-\frac{t_{f}-t_{0}}{2} \mathbf{f}\left(\mathbf{X}\left(\tau_{k}\right), \mathbf{U}\left(\tau_{k}\right)\right)=\mathbf{0}, \quad(k=1, \ldots, K)
$$

The approximated control, $\mathbf{U}(\boldsymbol{\tau})$, is defined in a similar manner as the state:

$$
\mathbf{u}(\tau) \approx \mathbf{U}(\tau)=\sum_{i=1}^{N} \mathcal{L}_{i}(\tau) \mathbf{U}\left(\tau_{i}\right)
$$

where $\tau_{i}, i=1, \ldots, N$ are again the LGL nodes. Again, we emphasize that in the LPM, the LGL points are both the discretization points and the collocation points. We will see in other methods that these two sets of points are not the same.

Next, the continuous cost function of Eq. (1) is approximated simply as

$$
J=\Phi\left(\mathbf{X}\left(\boldsymbol{\tau}_{N}\right)\right)
$$

Lastly, the boundary constraint of Eq. (3) is expressed as

$$
\boldsymbol{\phi}\left(\mathbf{X}\left(\tau_{1}\right), \mathbf{X}\left(\tau_{N}\right)\right)=\mathbf{0}
$$

The cost function of Eq. (18) and the algebraic constraints of Eqs. (16) and (19) define an NLP whose solution is an approximate solution to the continuous Mayer problem. Finally, it is noted that discontinuities in the state or control can be handled efficiently by dividing the trajectory into phases, where the dynamics are transcribed within each phase and then connected together by additional phase interface (a.k.a linkage) constraints. ${ }^{16}$

\subsection{Radau Pseudospectral Method (RPM)}

The Radau pseudospectral method has been primarily used in the chemical engineering community. The location of the nodes in the Radau method are based on the flipped Legendre-Gauss-Radau (LGR) points, which lie on the interval $\tau \in(-1,1]$. Recall that this set of points includes the final point but not the initial point. When discretizing optimal control problems, it is desirable that the discretization span the entire interval, including both endpoints. Therefore, in order to complete the full discretization of the time interval, the $\mathrm{N}$ discretization points are found using $N-1$ flipped Radau points plus the initial point, $\tau_{0} \equiv-1$. The state approximation is constructed exactly the same as the Legendre pseudospectral method, using a basis of N Lagrange polynomials:

$$
\mathbf{X}(\tau) \approx \mathbf{X}(\tau)=\sum_{i=0}^{N-1} \mathcal{L}_{i}(\tau) \mathbf{X}\left(\tau_{i}\right)
$$

where we make a slight index modification from $(i=1, \ldots, N)$ used in the LPM to $(i=0, \ldots, N-1)$. This new index highlights the fact that the nodes $\tau_{i}, i=0, \ldots, N-1$ are the initial point plus the $\mathrm{N}-1$ LGR points. 
Unlike the Legendre pseudospectral method, the Radau method uses a different number of collocation points, $K$, than discretization points, $N$. Specifically, the collocation points are the $N-1$ LGR points, while the discretization points are the LGR points plus the initial point, $\tau_{0}$. Therefore, $K=N-1$. The $K$ collocation equations are then described as

$$
\sum_{i=0}^{K} \dot{\mathcal{L}}_{i}\left(\tau_{k}\right) \mathbf{X}\left(\tau_{i}\right)-\frac{t_{f}-t_{0}}{2} \mathbf{f}\left(\mathbf{X}\left(\boldsymbol{\tau}_{k}\right), \mathbf{U}\left(\tau_{k}\right)\right)=\mathbf{0}, \quad(k=1, \ldots, K)
$$

where $\tau_{k}$ are the LGR points and

$$
\dot{\mathcal{L}}_{i}\left(\tau_{k}\right)=D_{k i}=\left\{\begin{array}{cl}
\frac{\dot{g}\left(\tau_{k}\right)}{\left(\tau_{k}-\tau_{i}\right) \dot{g}\left(\tau_{i}\right)}, & \text { if } k \neq i \\
\frac{\ddot{g}\left(\tau_{i}\right)}{2 \dot{g}\left(\tau_{i}\right)}, & \text { if } k=i
\end{array}\right.
$$

where the function $g\left(\boldsymbol{\tau}_{i}\right)=\left(1+\boldsymbol{\tau}_{i}\right)\left[P_{K}\left(\boldsymbol{\tau}_{i}\right)-P_{K-1}\left(\boldsymbol{\tau}_{i}\right)\right]$ and $\boldsymbol{\tau}_{i},(i=0, \ldots, K)$ are the $K$ LGR points plus the initial point. Since the collocation equations involve the control solely at the Radau points, the control is approximated using $N-1$ Lagrange polynomials, $\overline{\mathcal{L}}_{k}(\tau)(k=1, \ldots, N-1)$, as

$$
\mathbf{u}(\tau) \approx \mathbf{U}(\tau)=\sum_{k=1}^{N-1} \overline{\mathcal{L}}_{k}(\tau) \mathbf{U}\left(\tau_{k}\right)
$$

where $\tau_{k}$ again are the LGR points. This control approximation uses one less point than the state approximation meaning there is no control defined at the initial point. See Table 1 for more details. In practice, the initial control is simply extrapolated from Eq. (23). The discretization of the rest of the optimal control problem is similar to the LPM. The continuous cost function of Eq. (1) is approximated as

$$
J=\Phi\left(\mathbf{X}\left(\boldsymbol{\tau}_{K}\right)\right)
$$

Recall that in the Radau method, the final LGR point $\tau_{K}=1$. Lastly, the boundary constraint of Eq. (3) is expressed as a function of the initial point and the final point as

$$
\boldsymbol{\phi}\left(\mathbf{X}\left(\boldsymbol{\tau}_{0}\right), \mathbf{X}\left(\boldsymbol{\tau}_{K}\right)\right)=\mathbf{0}
$$

The cost function of Eq. (24) and the algebraic constraints of Eqs. (21) and (25) define an NLP whose solution is an approximate solution to the continuous Mayer problem. As with the LPM, discontinuities in the state or control can be handled efficiently by dividing the trajectory into phases. In fact, in the chemical engineering community, it is common practice to break the trajectory up into many phases and use relatively few numbers of collocation points in each phase. ${ }^{19}$

\subsection{Gauss Pseudospectral Method (GPM)}

The location of the nodes in the Gauss pseudospectral method are based on Legendre-Gauss (LG) points, which lie on the interval $\tau \in(-1,1)$. This set of points includes neither the initial point nor the final point. Therefore, in order to fully discretize the time interval, the $\mathrm{N}$ discretization points are $(N-2)$ interior LG points, the initial point, $\tau_{0} \equiv-1$, and the final point, $\tau_{F} \equiv 1$. The state is approximated using a basis of N-1 Lagrange interpolating polynomials, which is slightly smaller than the previous two methods,

$$
\mathbf{X}(\boldsymbol{\tau}) \approx \mathbf{X}(\boldsymbol{\tau})=\sum_{i=0}^{N-2} \overline{\mathcal{L}}_{i}(\tau) \mathbf{X}\left(\boldsymbol{\tau}_{i}\right)
$$


where $\tau_{i}(i=0, \ldots, N-2)$ are the initial point plus the $(N-2)$ LG points. Note that the final point, although it is part of the NLP discretization, is not part of the state approximation. This results in a state approximation that is one order less than the previous methods, but is necessary in order to have the equivalence property between the KKT conditions and HBVP conditions. This issue will be revisited in the results section.

As in the Radau pseudospectral method, the Gauss pseudospectral method uses a different number of collocation points, $K$, than discretization points, $N$. Specifically, the collocation points are the LG points, while the discretization points are the LG points plus the initial point and final point. Therefore, $K=N-2$. The $K$ collocation equations are then described as

$$
\sum_{i=0}^{K} D_{k i} \mathbf{X}\left(\tau_{i}\right)-\frac{t_{f}-t_{0}}{2} \mathbf{f}\left(\mathbf{X}\left(\tau_{k}\right), \mathbf{U}\left(\boldsymbol{\tau}_{k}\right)\right)=\mathbf{0}, \quad(k=1, \ldots, K)
$$

where $\tau_{k},(k=1, \ldots, K)$ are the LG points. Note that there is no collocation at either boundary. The differentiation matrix, $D \in \mathbb{R}^{K \times(N-1)}$, can be determined offline as follows:

$$
D_{k i}=\dot{\mathcal{L}}_{i}\left(\tau_{k}\right)=\left\{\begin{array}{cc}
\frac{\left(1+\tau_{k}\right) \dot{P}_{N}\left(\tau_{k}\right)+P_{N}\left(\tau_{k}\right)}{\left(\tau_{k}-\tau_{i}\right)\left[\left(1+\tau_{i}\right) \dot{P}_{N}\left(\tau_{i}\right)+P_{N}\left(\tau_{i}\right)\right]} & , \quad i \neq k \\
\frac{\left(1+\tau_{i}\right) \ddot{P}_{N}\left(\tau_{i}\right)+2 \dot{P}_{N}\left(\tau_{i}\right)}{2\left[\left(1+\tau_{i}\right) \dot{P}_{N}\left(\tau_{i}\right)+P_{N}\left(\tau_{i}\right)\right]} & , i=k
\end{array}\right.
$$

where $\tau_{k},(k=1, \ldots, K)$ are the LG points and $\tau_{i},(i=0, \ldots, K)$ are the LG points plus the initial point. The control is approximated at the $(N-2)$ collocation points using a basis of $(N-2)$ Lagrange interpolating polynomials $\tilde{\mathcal{L}}_{i}(\tau),(i=1, \ldots, N-2)$ as

$$
\mathbf{u}(\boldsymbol{\tau}) \approx \mathbf{U}(\boldsymbol{\tau})=\sum_{i=1}^{N-2} \tilde{\mathcal{L}}_{i}(\boldsymbol{\tau}) \mathbf{U}\left(\boldsymbol{\tau}_{i}\right)
$$

where $\tau_{i},(k=1, \ldots, N-2)$ are the LG points. Table 1 lists all the Lagrange polynomial expressions described in this work. Close inspection of Eq. (27) reveals that the collocation equations are independent of the final state, $\mathbf{X}\left(\boldsymbol{\tau}_{F}\right)$. Therefore, an additional constraint must be added to the discretization in order to ensure that the the final state obeys the state dynamics. This is enforced by including a Gauss quadrature to approximate the integral of the dynamics across the entire interval:

$$
\mathbf{X}\left(\boldsymbol{\tau}_{F}\right)-\mathbf{X}\left(\boldsymbol{\tau}_{0}\right)-\frac{t_{f}-t_{0}}{2} \sum_{k=1}^{K} w_{k} \mathbf{f}\left(\mathbf{X}\left(\tau_{k}\right), \mathbf{U}\left(\tau_{k}\right)\right)=\mathbf{0}
$$

where $w_{k}$ are the Gauss weights and $\tau_{k}$ are the LG points. Lastly, the cost function is approximated by

$$
J=\Phi\left(\mathbf{X}\left(\boldsymbol{\tau}_{F}\right)\right)
$$

and the boundary constraint is expressed as

$$
\boldsymbol{\phi}\left(\mathbf{X}\left(\boldsymbol{\tau}_{0}\right), \mathbf{X}\left(\boldsymbol{\tau}_{F}\right)\right)=\mathbf{0}
$$

The cost function of Eq. (31) and the algebraic constraints of Eqs. (27), (30), and (32) define an NLP whose solution is an approximate solution to the continuous Mayer problem. The handling of discontinuities in the state or control is explained in the previous two methods. 


\section{Costate Estimate}

All three aforementioned pseudospectral methods have an established costate estimation procedure. In particular, a mapping between the KKT multipliers of the NLP and the costates of the continuoustime optimal control problem has been derived for all three methods. In this section we describe these costate estimation procedures which will be implemented later in the paper.

\subsection{Legendre Pseudospectral Method}

The KKT conditions of the NLP are derived by defining an augmented cost function, which brings the NLP constraints into the cost function via Lagrange multipliers. For LPM, the augmented cost is

$$
J_{a}=\boldsymbol{\Phi}\left(\mathbf{X}_{N}\right)-\tilde{\boldsymbol{v}}^{T} \boldsymbol{\phi}\left(\mathbf{X}_{1}, \mathbf{X}_{N}\right)-\sum_{k=1}^{N} \tilde{\Lambda}_{k}^{T}\left(\sum_{i=1}^{N} D_{k i} \mathbf{X}_{i}-\frac{t_{f}-t_{0}}{2} \mathbf{f}\left(\mathbf{X}_{k}, \mathbf{U}_{k}\right)\right)
$$

The KKT multipliers in Eq. (33) are $\tilde{\Lambda}_{k},(k=1, \ldots, K)$, which are associated with the collocation equations of Eq. (16) and $\tilde{\boldsymbol{v}}$, which relates to the boundary condition of Eq. (19). The mapping from the KKT multipliers to the HBVP variables is: ${ }^{25}$

$$
\begin{aligned}
\Lambda_{k} & =\frac{\tilde{\Lambda}_{k}}{w_{k}}, \quad(k=1, \ldots, K) \\
\boldsymbol{v} & =\tilde{\boldsymbol{v}}
\end{aligned}
$$

The HBVP variables $\Lambda_{k},(k=1, \ldots, N)$, and $\boldsymbol{v}$, now defined in terms of NLP multipliers, can be substituted into the HBVP equations to determine if the optimality conditions have been met. It has been documented in the literature ${ }^{26,28}$ that this mapping for the LPM does not provide an exact equivalence between the KKT conditions and the HBVP conditions. Specifically, the HBVP costate differential equations are not naturally collocated at the boundaries. In other words, the boundary KKT conditions result in a linear combination of two HBVP conditions: the boundary costate dynamics and the transversality conditions. We will not derive all the KKT conditions here, but we will present the conflicting KKT conditions, which is found by taking the partial derivative of the augmented cost function with respect to the initial state:

$$
\sum_{k=1}^{N} \Lambda_{k} D_{1 k}+\frac{t_{f}-t_{0}}{2} \Lambda_{1} \frac{\partial \mathbf{f}_{1}}{\partial \mathbf{X}_{1}}=\frac{1}{w_{1}}\left(-\Lambda_{1}+\boldsymbol{v}^{T} \frac{\partial \boldsymbol{\phi}}{\partial \mathbf{X}_{1}}\right)
$$

Notice that the left-hand side of the equation resembles the discretized costate dynamics and the right hand side resembles the transversality condition from the HBVP in Section 3. In order to map these conditions to the HBVP equations, each side of Eq. (35) should equal zero. However, the two conditions are inseparable in the LPM. Similarly, the KKT condition corresponding to the final state is

$$
\sum_{k=1}^{N} \Lambda_{k} D_{N k}+\frac{t_{f}-t_{0}}{2} \Lambda_{N} \frac{\partial \mathbf{f}_{N}}{\partial \mathbf{X}_{N}}=\frac{1}{w_{N}}\left(\Lambda_{N}-\frac{\partial \boldsymbol{\Phi}}{\partial \mathbf{X}_{N}}+\boldsymbol{v}^{T} \frac{\partial \boldsymbol{\phi}}{\partial \mathbf{X}_{N}}\right)
$$

where again, the left-hand side is the discretized costate dynamics and the right-hand side is the transversality condition corresponding to the terminal state. It is impossible to get an exact mapping from the KKT conditions to the HBVP conditions without being able to separate these mixed conditions. Recent research ${ }^{26}$ suggests that by applying relaxation techniques, linear independence 
constraint qualification, and an additional set of conditions called "closure conditions", one can show that there exists a costate approximation that converges to the true costate in the limit. From Ref. 26, the most recent closure conditions are

$$
\| \begin{aligned}
& -\Lambda_{1}^{*}-\frac{\partial \Phi}{\partial \mathbf{x}_{1}}\left(\mathbf{X}_{1}^{*}, \mathbf{X}_{N}^{*}\right)-\left(\frac{\partial \phi}{\partial \mathbf{x}_{1}}\left(\mathbf{X}_{1}^{*}, \mathbf{X}_{N}^{*}\right)\right)^{T} v^{*} \|_{\infty} \leq \delta_{D} \\
& \Lambda_{N}^{*}-\frac{\partial \Phi}{\partial \mathbf{x}_{N}}\left(\mathbf{X}_{1}^{*}, \mathbf{X}_{N}^{*}\right)-\left(\frac{\partial \phi}{\partial \mathbf{x}_{N}}\left(\mathbf{X}_{1}^{*}, \mathbf{X}_{N}^{*}\right)\right)^{T} v^{*} \|_{\infty} \leq \delta_{D}
\end{aligned}
$$

where $\mathbf{X}^{*}, \Lambda^{*}$, and $\boldsymbol{v}^{*}$ represent a solution that satisfies Eq. (37) and the KKT conditions, and $\delta_{D}$ is a dual feasibility tolerance. These conditions are essentially the discretized HBVP transversality conditions. ${ }^{1}$ However, it is unclear how the closure conditions are implemented. The covector mapping theorem ${ }^{26}$ states that there exists an $N$ large enough so that the closure conditions and the KKT conditions will be satisfied to a specified tolerance, $\delta$. Upon close examination of Ref. 26 (and the references therein) any algorithm attempting to solve the combined KKT conditions and closure conditions must solve a mixed primal-dual feasibility problem. By including the closure conditions of Eq. (37) to form the "augmented optimality conditions", both the state and costate are now variables to be solved. This procedure significantly increases the computational complexity in finding a costate approximation. However, both LPM costate approximations (Refs. 25 and 26) are considered in this paper and their accuracies are compared with the Radau and Gauss methods in the results section. The LPM costate will be computed first according to Eq. (34), and then by formulating the primal-dual feasibility problem which attempts to satisfy the augmented KKT conditions according to some tolerance, $\delta$. It is noted that improving the LPM costate approximation is an ongoing research topic, and better methods may be found in the future.

Similar to the LPM state approximate, the continuous-time approximation for the costate is represented by a basis of $N$ Lagrange interpolating polynomials as

$$
\boldsymbol{\lambda}(\tau) \approx \Lambda(\tau)=\sum_{i=1}^{N} \Lambda\left(\tau_{i}\right) \mathcal{L}_{i}(\tau)
$$

\subsection{Radau Pseudospectral Method}

The augmented cost function corresponding to the Radau pseudospectral method is given as

$$
J_{a}=\boldsymbol{\Phi}\left(\mathbf{X}_{K}\right)-\tilde{\boldsymbol{v}}^{T} \boldsymbol{\phi}\left(\mathbf{X}_{0}, \mathbf{X}_{K}\right)-\sum_{k=1}^{K} \tilde{\Lambda}_{k}^{T}\left(\sum_{i=0}^{K} D_{k i} \mathbf{X}_{i}-\frac{t_{f}-t_{0}}{2} \mathbf{f}\left(\mathbf{X}_{k}, \mathbf{U}_{k}\right)\right)
$$

The KKT multipliers resulting from the NLP using the Radau pseudospectral method are $\tilde{\Lambda}_{k}, \quad(k=$ $1, \ldots, K)$, associated with the collocation equations of Eq. (21) and $\tilde{\boldsymbol{v}}$, associated with the boundary conditions of Eq. (25). The mapping from the KKT multipliers to the HBVP variables is described as follows:

$$
\begin{aligned}
\Lambda_{k} & =\frac{\tilde{\Lambda}_{k}}{w_{k}}, \quad(k=1, \ldots, K) \\
\boldsymbol{v} & =\tilde{\boldsymbol{v}}
\end{aligned}
$$

The $K$ discrete costates $\Lambda_{k},(k=1, \ldots, K)$, and the HBVP multiplier $v$ are now defined in terms of the KKT multipliers. As in the LPM, it is understood that there is an imperfect match between the KKT 
multipliers and the costate. ${ }^{21}$ The Radau method has a similar conflict of HBVP equations when the partial derivative of the augmented cost function is taken with respect to the final state:

$$
\sum_{k=1}^{N} \Lambda_{k} D_{K k}+\frac{t_{f}-t_{0}}{2} \Lambda_{K} \frac{\partial \mathbf{f}_{K}}{\partial \mathbf{X}_{K}}=\frac{1}{w_{K}}\left(\Lambda_{K}-\frac{\partial \Phi}{\partial \mathbf{X}_{K}}+\boldsymbol{v}^{T} \frac{\partial \boldsymbol{\phi}}{\partial \mathbf{X}_{K}}\right)
$$

However, recent work has demonstrated rapid convergence rates of the costate approximation using Eq. (40) as the number of nodes increases. The discrete values of the costate are used to construct a continuous-time approximation to the costate, which is represented by a basis of $N-1$ Lagrange interpolating polynomials, as in the control: ${ }^{21}$

$$
\lambda(\tau) \approx \Lambda(\tau)=\sum_{i=1}^{K} \Lambda\left(\tau_{i}\right) \mathcal{L}_{i}(\tau)
$$

where $\tau_{k},(k=1, \ldots, K)$ are the LGR points. Note that since there is no collocation at the initial point, there is no initial costate estimate that is directly output from the NLP. However, the initial costate can either be computed by extrapolating Eq. (42) or by deriving the transversality conditions and using the initial state from the NLP to solve for the initial costate. Naturally, the former method is computationally simpler and is just as accurate.

\subsection{Gauss Pseudospectral Method}

The augmented cost function corresponding to the Gauss discretization is

$$
\begin{aligned}
J_{a}= & \boldsymbol{\Phi}\left(\mathbf{X}_{F}\right)-\tilde{\boldsymbol{v}}^{T} \boldsymbol{\phi}\left(\mathbf{X}_{0}, \mathbf{X}_{F}\right)-\sum_{k=1}^{K} \tilde{\Lambda}_{k}^{T}\left(\sum_{i=0}^{K} D_{k i} \mathbf{X}_{i}-\frac{t_{f}-t_{0}}{2} \mathbf{f}\left(\mathbf{X}_{k}, \mathbf{U}_{k}\right)\right) \\
& -\tilde{\Lambda}_{F}^{T}\left(\mathbf{X}_{F}-\mathbf{X}_{0}-\frac{t_{f}-t_{0}}{2} \sum_{k=1}^{K} w_{k} \mathbf{f}\left(\mathbf{X}_{k}, \mathbf{U}_{k}\right)\right)
\end{aligned}
$$

The KKT multipliers resulting from the NLP using the Gauss pseudospectral method are $\tilde{\Lambda}_{k}(k=$ $1, \ldots, K)$, associated with the collocation equations of Eq. (27), $\tilde{\Lambda}_{F}$, associated with the quadrature constraint of Eq. (30), and $\tilde{\boldsymbol{v}}$, associated with the boundary conditions of Eq. (32). The mapping from the KKT multipliers to the HBVP variables is described as follows:

$$
\begin{aligned}
\boldsymbol{\Lambda}_{k} & =\frac{\tilde{\Lambda}_{k}}{w_{k}}+\tilde{\Lambda}_{F}, \quad(k=1, \ldots, K) \\
\boldsymbol{v} & =\tilde{\boldsymbol{v}} \\
\Lambda_{f} & =\tilde{\Lambda}_{F}
\end{aligned}
$$

The variables $\Lambda_{k}, \Lambda_{f}$, and $\boldsymbol{v}$, now defined in terms of NLP multipliers, can be substituted into the HBVP equations to ensure the optimality conditions have been met. The discrete costate is then used to construct a continuous-time costate approximation, which is represented by a slightly different basis of $N-1$ Lagrange interpolating polynomials, defined as

$$
\boldsymbol{\lambda}(\tau) \approx \Lambda(\tau)=\sum_{i=1}^{K+1} \Lambda\left(\tau_{i}\right) \overline{\mathcal{L}}_{i}(\tau)
$$


where $\boldsymbol{\tau}_{i},(i=1, \ldots, K+1)$ are the LG points plus the final point, $\boldsymbol{\tau}_{F}$. Recall that the state is approximated using $(N-1)$ Lagrange polynomials based on the LG points and the initial time, while the costate, $\boldsymbol{\lambda}(\boldsymbol{\tau})$, is approximated using $(N-1)$ Lagrange polynomials consisting of the LG points and the final time. This discrepancy is required to preserve the mapping, and the reader can find more detailed information in Ref. 24. As in the Radau method, an approximation for the initial costate, $\Lambda_{0}$, cannot be pulled directly from a corresponding multiplier in the NLP because no such multiplier exists. However, an accurate approximation to the initial costate can be determined from the following equation:

$$
\Lambda_{0} \equiv \tilde{\Lambda}_{F}-\sum_{k=1}^{K} D_{k 0} \tilde{\Lambda}_{k} \quad(k=1, \ldots, K)
$$

It is shown in Ref. 38 that this map for the initial costate also satisfies the HBVP transversality condition

$$
\Lambda_{0}=\boldsymbol{v}^{T} \frac{\partial \boldsymbol{\phi}}{\partial \mathbf{X}_{0}}
$$

resulting in a perfect match between the HBVP equations and the KKT conditions. Furthermore, one could simply extrapolate Eq. (45) to $\tau_{0}$, which would produce an equally accurate initial costate. All three methods for determining the initial costate are equivalent. The remainder of this paper focuses on comparing these pseudospectral techniques in terms of their computational efficiency, approximation accuracy, and solution convergence rates with respect to the state, control, and especially the costate.

\section{Single State Example}

As a first example, we consider the following one-dimensional optimal control problem. Minimize the cost functional

$$
J=-y\left(t_{f}\right)
$$

subject to the constraints

$$
\dot{y}(t)=y(t) u(t)-y(t)-u^{2}(t)
$$

and the initial condition

$$
y\left(t_{0}\right)=1
$$

where $y(t)$, is the state, $u(t)$ is the control, and $t_{f}=5$ is the final time. The exact solution is

$$
\begin{aligned}
y^{*}(t) & =\frac{4}{1+3 e^{t}} \\
\lambda^{*}(t) & =a e^{\left(2 \ln \left(1+3 e^{t}\right)-t\right)} \\
u^{*}(t) & =.5 y^{*}(t) \\
\text { where } a & =\frac{-1}{\left(e^{-5}+6+9 e^{5}\right)}
\end{aligned}
$$

$\lambda^{*}(t)$ is the costate associated with the optimal solution. The exact solution is depicted in Fig. 2. This problem is solved using all three pseudospectral techniques mentioned previously. Each method transcribes the optimal control problem into an NLP that consists of 10 nodes $(N=10)$. Each NLP is then solved with SNOPT ${ }^{39}$ using the default feasibility and optimality tolerances $\left(10^{-6}\right)$. In Fig. 3, the absolute value of the error between the NLP state and the exact solution $\left(\left|Y\left(\tau_{i}\right)-y^{*}\left(\tau_{i}\right)\right|, i=1, \ldots, N\right)$ 
is plotted for each node and all three approaches. Note that the error in initial state is not included since the SNOPT satisfies state equality bounds (i. e. the initial conditions) to double precision $\left(10^{-16}\right)$. When comparing the state accuracy of the three methods in this example, there is no clear method that outperforms the other two across the entire interval. In fact, the only noticeable difference in Fig. 3 is that both the GPM and Radau formulations produce an extremely accurate terminal state. It is hypothesized that the highly accurate terminal state for the GPM is due to the additional quadrature constraint in the NLP.

The control error is plotted in Fig. 4. As described in Section 5, each method approximates the control using a different number of points. The LPM discretizes the control at all $\mathrm{N}$ discretization points, while Radau discretizes the control at the (N-1) Radau points, and the Gauss method discretizes the control at the (N-2) Gauss points. In the Radau discretization, the initial control is missing. Similarly, in the Gauss discretization, the control at both the initial and final time are missing. These missing control values can be found by a variety of techniques. The approach often outlined in the literature $^{6,19}$ is to extrapolate the control approximation equations, Eqs. (17), (23) and (29). However, in practice it is more common to use a simple spline extrapolation. ${ }^{40}$ Thirdly, a technique involving the HBVP equations at the boundaries has been developed for the Gauss pseudospectral method. ${ }^{41}$ Since these extrapolated controls are not explicit variables in the NLP, one would assume that they would be less accurate than methods that include the boundary controls in the formulation of the NLP. In Fig. 4, the boundary control estimate for the Gauss method is indeed the least accurate. Likewise, the accuracy of the initial control estimate for the Radau method is similar to the GPM value. However, it is interesting that while the largest error for Gauss and Radau are at the boundaries, the LPM has a larger error at one of the interior points. The benefit of an accurate initial control is eliminated by a very inaccurate interior point in the LPM.

A more appropriate way to determine the effect of the control error is to actually propagate the state dynamics according to the control estimate from the NLP. In this research, the state dynamics are propagated using the Matlab function ODE45, where the control approximations are represented using Eqs. (17), (23), and (29). The resulting propagated state error is shown in Fig. 5. Clearly, the LPM propagation error is the largest despite the relatively accurate boundary controls.

The largest discrepancy between the methods is seen in the costate comparison of Fig. 6. The LPM costate approximation is several orders of magnitude worse than the other two methods across all nodes. This example highlights a common problem ${ }^{26}$ with the original LPM costate approximation ${ }^{25}$ where the approximation tends to "wiggle" about the true solution, as shown in Fig. 7. Furthermore, note that the largest error in the LPM costate occurs at the boundaries due to the conflicting constraints (explained in Section 6.1). The GPM method, which has no constraint conflict, provides extremely accurate boundary costates, while the RPM method produces an accurate initial costate (no conflict) but a less accurate final costate (which has a conflict explained in Section 6.2).

In pseudospectral methods, like most direct methods, the solution accuracy can be improved by increasing the number of nodes used to discretize the problem. ${ }^{11}$ Furthermore, the rate of convergence to the true optimal solution is extremely important as it can help determine how many nodes are needed in order to adequately approximate the solution. The convergence rates of the state and costate are shown in Figs. 8-9, respectively. In these figures, each method was solved repeatedly while the number of nodes in the problem was increased from 5 to 50 . The error shown in these figures is the maximum absolute error over all the nodes in the problem $\left(\left\|X\left(\boldsymbol{T}_{i}\right)-x^{*}\left(\boldsymbol{\tau}_{i}\right)\right\|_{\infty}, \forall \boldsymbol{\tau}_{i} \in[-1,1]\right)$. As seen in Figure 8, the state convergence rate for all three methods is quite similar. The steep convergence initially seen depicts the "spectral" convergence that is characteristic of pseudospectral methods. ${ }^{8}$ Naturally, once the error drops below the tolerances of the NLP, the convergence rate stops improving. The convergence rate for the costate is shown in Fig. 9. The Gauss and Radau methods show rapid convergence rates for the costate, which are even faster than the state. Fig. 9 also shows 
the apparent lack of convergence for the costate using the LPM. It is clear that increasing the number of nodes does not improve the costate error for this example. As mentioned previously, Gong and Ross have devised a modified covector mapping theorem to improve the costate approximation for the LPM. This covector mapping theorem includes a set of "closure conditions" that is assumed to be implemented in a mixed primal-dual feasibility problem where the variables are the state, control, and costate. The constraints in this primal-dual feasibility problem are the KKT conditions of the NLP and the additional closure conditions. When this alternate NLP is posed and solved, the resulting costate convergence in Fig. 10 is indeed much better, although it is still not as rapid as either the Gauss or Radau methods. As mentioned previously, this increased accuracy comes at a significant computational burden in post-processing. Due to the large number of extra steps necessary to produce this result, further computation of LPM costates will involve only the original costate estimation procedure of Eq. (34), since this is the likely method to be used in practice.

\section{Two State Example}

As a second example, consider the following two-dimensional optimal control problem. Minimize the cost functional

$$
J=y_{2}\left(t_{f}\right)
$$

subject to the dynamic constraints

$$
\begin{aligned}
& \dot{y}_{1}(t)=0.5 y_{1}(t)+u(t) \\
& \dot{y}_{2}(t)=y_{1}^{2}(t)+0.5 u^{2}(t)
\end{aligned}
$$

and the boundary conditions,

$$
\begin{aligned}
& y_{1}\left(t_{0}\right)=1 \quad y_{1}\left(t_{f}\right)=0.5 \\
& y_{2}\left(t_{0}\right)=0
\end{aligned}
$$

where $t_{f}=5$. Note that this problem contains a terminal bound on the first state, $y_{1}\left(t_{f}\right)$. The exact solution is of the form

$$
\begin{aligned}
& y_{1}^{*}(t)=a_{1} e^{1.5 t}+a_{2} e^{-1.5 t}, \\
& y_{2}^{*}(t)=a_{3}\left(e^{1.5 t}\right)^{2}-a_{4}\left(e^{-1.5 t}\right)^{2}+c_{1} \\
& \lambda_{1}^{*}(t)=a_{5} e^{1.5 t}+a_{6} e^{-1.5 t}, \\
& \lambda_{2}^{*}(t)=1 \\
& u^{*}(t)=-\lambda_{1}^{*}(t)
\end{aligned}
$$

The exact solution can be seen in Fig. 11. Again, all three methods are compared using 10 nodes. The error plots are formulated in the same manner as the previous example problem. Fig. 12 shows the state error at each node for all three methods. Recall that the error for the states involving the boundary conditions is $O\left(10^{-16}\right)$, and therefore outside the range of the plot. As in the single-state example, all three methods are relatively comparable in state accuracy. Moreover, the GPM and Radau formulations show again show an increase in accuracy for the terminal state.

The control error is plotted in Fig. 13. Interestingly, for this problem, the LPM control estimate is worse than both GPM and RPM, even at the boundaries. The NLP control is used to propagate the state equations, and their resulting state accuracy is compared in Fig. 14 for both states. In this example, the RPM produces the most accurate approximation, followed by the GPM, and lastly LPM. 
Reconfirming the results in the first example, the largest discrepancy between the methods is seen in the costate comparison, and specifically with the second costate, shown in Fig. 15. The LPM method produces the worst costate approximation for both $\lambda_{1}$ and $\lambda_{2}$. Although it is not shown, this large error in $\lambda_{2}$ is due to the same "wiggle" phenomenon around the true costate as seen in the first example. The GPM method also provides the most accurate boundary costates, and the Radau method provides a relatively accurate initial costate, but an inaccurate final costate. In the Radau method, the final costate is consistently the least accurate costate, suggesting that the conflicting HBVP equations described in Section 6.2 is degrading the performance of this method.

The convergence rates of the state and costate are shown in Figs. 16-17, respectively. Each approach was solved repeatedly while the number of nodes in the problem was increased from 5 to 30 . The error shown in these figures is computed in the same manner as the previous example. Fig. 16 displays both states, $y_{1}$ and $y_{2}$, where there is a noticeable difference between the convergence rate of $y_{1}$ and $y_{2}$ for the GPM and RPM. However, for the LPM approach, both states converge at the same (slower) rate. The convergence rate of the RPM is the fastest for this example. It is hypothesized that this slight improvement over the GPM state approximation is due to the one order reduction in the state approximation for the GPM. Similar results are seen in the costate convergence plot of Fig. 17. The second costate is constant in this example, and both the GPM and RPM approaches can approximate this first order polynomial quite well, even using only a few nodes, as expected with pseudospectral methods. However, the LPM approach displays the wiggle phenomenon and thus has a much slower convergence rate for $\lambda_{2}$. In term of the first costate, the GPM has the fastest convergence rate.

\section{Orbit-Raising Problem}

Consider the problem of transferring a spacecraft from an initial circular orbit to the largest possible circular orbit in a fixed time using a low-thrust engine. This problem is modeled using radius, $r(t)$, radial velocity, $u(t)$, and tangential velocity, $v(t)$, as the components of the state. The control, $\phi(t)$, is the angle between the thrust vector and the tangential velocity. The optimal control problem is stated as follows. Minimize the cost functional

$$
J=-r\left(t_{f}\right)
$$

subject to the dynamic constraints

$$
\begin{aligned}
\dot{r} & =u \\
\dot{u} & =\frac{v^{2}}{r}-\frac{\mu}{r^{2}}+\frac{T \sin \phi}{m_{0}-|\dot{m}| t} \\
\dot{v} & =-\frac{u v}{r}+\frac{T \cos \phi}{m_{0}-|\dot{m}| t}
\end{aligned}
$$

and the boundary conditions

$$
\begin{array}{lll}
r(0)=1, & r\left(t_{f}\right)=\text { free } \\
u(0)=0, & u\left(t_{f}\right)=0, \\
v(0)=\sqrt{\frac{\mu}{r(0)}}, & v\left(t_{f}\right)=\sqrt{\frac{\mu}{r\left(t_{f}\right)}}
\end{array}
$$

where $\mu$ is the gravitational parameter, $T$ is the thrust magnitude, $m_{0}$ is the initial mass, and $\dot{m}$ is the fuel consumption rate. These parameters are given as follows in normalized units:

$$
T=0.1405, \quad \dot{m}=0.0749, \quad m_{0}=\mu=1, \quad t_{f}=3.32
$$


This problem does not have an analytic solution, but it has been solved numerically many times ${ }^{38,42}$ so it's solution is well known. The results of each method was compared against the solution to a boundary value problem solution using the Matlab function BVP4C with a tolerance of $10^{-9}$. For brevity, we present an error analysis for only the first state and costate, $r(t)$ and $\lambda_{r}(t)$. The remaining state and costate errors look very similar to the one presented. For this problem, the RPM has a slightly better state accuracy than the other two methods in Fig. 18. Interestingly, for this example, the RPM also has a slightly better costate approximation than the other two methods as seen in Fig. 19. As in the previous examples, the LPM costate convergence is significantly worse than the other two methods.

Based on the results of all three example problems and a detailed analysis of the mathematics of each method, we can conclude that there may be certain circumstances under which each method should be chosen. Inaccurate boundary costates in the LPM method are attributed to boundary costs or constraints in the problem. However, if the original problem has no boundary constraints or costs, then the conflicting HBVP equations disappear and LPM would be an appropriate method. The RPM costate inaccuracy only occurs at the terminal time, so problems without terminal constraints would likely mitigate the errors in the final costate. The GPM has a perfect mapping with the HBVP equations, and therefore will create accurate costate estimates for problems with both initial and final constraints.

Lastly, Table 2 presents NLP computation times for all three examples and all three methods. The results listed correspond to a Pentium 4, $3.2 \mathrm{GHz}$ machine, where the constraint Jacobian was computed using numerical derivatives. All three methods have very similar computation times, which is expected since the methods are very similar in terms of their problem density.

\section{Conclusions}

A comparison has been made between three established pseudospectral methods that have been used in recent years in the numerical solution of optimal control problems. In particular, the Legendre, Radau, and Gauss pseudospectral methods have been compared in terms of the accuracy of the state, control, and costate. Three examples are used in the study to identify the key differences between the three methods. The results of this study indicate that the accuracy of the Radau and Gauss methods are very similar in accuracy, while both of these methods significantly outperform the Legendre method in terms of costate accuracy. Furthermore, it is found that the computational efficiency of the three methods is quite comparable. Based on these results and a detailed analysis of the mathematics of each method, a rationale has been constructed to determine when each method should be chosen.

\section{References}

${ }^{1}$ Kirk, D. E. , Optimal Control Theory, Prentice-Hall, Englewood Cliffs, NJ, 1970.

${ }^{2}$ Pontryagin, L.S., Boltyanskii, V., Gamkrelidze, R., Mischenko, E., The Mathematical Theory of Optimal Processes. New York: Interscience, 1962.

${ }^{3}$ Betts, John T., "Survey of Numerical Methods for Trajectory Optimization," Journal of Guidance, Control, and Dynamics, Vol. 21, No. 2, March-April 1998

${ }^{4}$ Hull, D. , "Conversion of Optimal Control Problems into Parameter Optimization Problems," AIAA Guidance, Navigation, and Control Conference, San Diego, CA, July 29-31, 1996 
${ }^{5}$ Vasantharajan, S. , and Biegler, L. T. , "Simultaneous Strategies for Optimization of DifferentialAlgebraic Systems with Enforcement of Error Criteria," Computers and Chemical Engineering, Vol. 14, No. 10, pp.1083-1100, 1990.

${ }^{6}$ Elnagar, G., Kazemi, M., Razzaghi, M., “The Pseudospectral Legendre Method for Discretizing Optimal Control Problems," IEEE Transactions on Automatic Control, Vol. 40, No. 10, October 1995

${ }^{7}$ Canuto, C., Hussaini, M.Y., Quarteroni, A., Zang, T.A., Spectral Methods in Fluid Dynamics, SpringerVerlag, New York, 1988.

${ }^{8}$ Fornberg, B., A Practical Guide to Pseudospectral Methods, Cambridge University Press, 1998.

${ }^{9}$ Villadsen, J. , and Michelsen, M. , "Solution of differential equation models by polynomial approximation,” Prentice Hall, New Jersey, 1978.

${ }^{10}$ Cuthrell, J. E. , and Biegler, L. T. , "On the Optimization of DIfferential-Algebraic Process Systems," AIChE Journal, Vol.33, No.8, pp.1257-1270, Aug. 1987.

${ }^{11}$ Trefethen, L. N., Spectral Methods in MATLAB, SIAM Press, Philadelphia, 2000.

${ }^{12}$ Elnagar, G. E. , and Kazemi, M. A. , "Pseudospectral Legendre-based optimal computation of nonlinear constrained variational problems," Journal of Computational and Applied Mathematics, Vol. 88, pp.363-375, 1997.

${ }^{13}$ Fahroo, F. , and Ross, I. M. , “A Spectral Patching Method for Direct Trajectory Optimization,” Journal of the Astronautical Sciences, Vol. 48, No. 2-3, pp.269-286, Apr-Sept 2000.

${ }^{14}$ Ross, I. M. , and Fahroo, F. , “A Direct Method for Solving Nonsmooth Optimal Control Problems”, Proceedings of the 2002 World Congress of the International Federation on Automatic Control, IIAC, Barcelona, July 2002.

${ }^{15}$ Rao, A. V. , "Extension of a Pseudospectral Legendre Method to Non-Sequential Multiple-Phase Optimal Control Problems”, AIAA Guidance, Navigation, and Control Conference, AIAA Paper No. 20035634, Austin, TX, Aug. 11-14, 2003.

${ }^{16}$ Ross, I. M. , and Fahroo, F. , "Pseudospectral Knotting Methods for Solving Optimal Control Problems," Journal of Guidance, Control, and Dynamics, Vol. 27, No. 3, 2004.

${ }^{17}$ Cuthrell, J. E. , and Biegler, L. T. , "Simultaneous Optimization and Solution Methods for Batch Reactor Control Profiles," Computers and Chemical Engineering, Vol. 13, No.1/2, pp.49-62, 1989.

${ }^{18}$ Reddien, G. W. , “Collocation at Gauss Points as a Discretization in Optimal Control,” SIAM Journal of Control and Optimization, Vol. 17, No. 2, March 1979.

${ }^{19}$ Kameswaran, S. and Biegler, L. T., "Convergence Rates for Dynamic Optimization Using Radau Collocation," SIAM Conference on Optimization, Stockholm, Sweden, 2005.

${ }^{20}$ Fahroo, F. and Ross, I., "Pseudospectral Methods for Infinite Horizon Nonlinear Optimal Control Problems," 2005 AIAA Guidance, Navigation, and Control Conference, AIAA Paper 2005-6076, San Francisco, CA, August 15-18, 2005.

${ }^{21}$ Kameswaran, S. and Biegler, L. T., "Convergence Rates for Direct Transcription of Optimal Control Problems at Radau Points," Proceedings of the 2006 American Control Conference, Minneapolis, Minnesota, June 2006. 
${ }^{22}$ Vlassenbroeck, J. and Van Doreen, R., "A Chebyshev Technique for Solving Nonlinear Optimal Control Problems," IEEE Transactions on Automatic Control, Vol. 33, No. 4, 1988, pp. 333-340.

${ }^{23}$ Vlassenbroeck, J., "A Chebyshev Polynomial Method for Optimal Control with State Constraints," Automatica, Vol. 24, 1988, pp. 499-506.

${ }^{24}$ Benson, D. A., Huntington, G. T., Thorvaldsen, T. P., and Rao, A. V., Direct Trajectory Optimization and Costate Estimation via an Orthogonal Collocation Method, Journal of Guidance, Control, and Dynamics, Vol. 29, No. 6, 2006, pp. 1435-1440.

${ }^{25}$ Fahroo, F. and Ross, I. M., "Costate Estimation by a Legendre Pseudospectral Method," Journal of Guidance, Control, and Dynamics, Vol. 24, No. 2, March-April 2002, pp. 270-277.

${ }^{26}$ Gong, Q. , Ross, I. , Kang, W. , and Fahroo, F. , "On the Pseudospectral Covector Mapping Theorem for Nonlinear Optimal Control,” IEEE Conference on Decision and Control, Dec 13-15, 2006.

${ }^{27}$ Williams, P. , “Jacobi Pseudospectral Method for Solving Optimal Control Problems”, Journal of Guidance, Vol. 27, No. 2,2003

${ }^{28}$ Williams, P. , "Hermite-Legendre-Gauss-Lobatto Direct Transcription Methods In Trajectory Optimization," Advances in the Astronautical Sciences. Vol. 120, Part I, pp. 465-484. 2005

${ }^{29}$ Williams, P. , “A Comparison of Differentiation and Integration Based Direct Transcription Methods", AAS/AIAA Space Flight Mechanics Conference, Copper Mountain, CO, Jan. 23-27, 2005. Paper AAS 05-128.

${ }^{30}$ Fahroo, F. and Ross, I. M., "Direct Trajectory Optimization by a Chebyshev Pseudospectral Method," Journal of Guidance, Control, and Dynamics, Vol. 25, No. 1, January-February 2002, pp. 160-166.

${ }^{31}$ Gong, Q. , Kang, W. , and Ross, I. , “A Pseudospectral Method for the Optimal Control of Constrained Feedback Linearizable Systems”, IEEE Transactions on Automatic Control, Vol. 51, No. 7, July 2006.

${ }^{32}$ Paris, S. W. , Riehl, J. P. , Sjuaw, W. .K. , "Enhanced Procedures for Direct Trajectory Optimization Using Nonlinear Programming and Implicit Integration,” AIAA/AAS Astrodynamics Specialist Conference and Exhibit, Keystone CO, Aug 2006

${ }^{33}$ Fahroo, F. , Ross, I. M. , “On Discrete-Time Optimality Conditions for Pseudospectral Methods,” AIAA/AAS Astrodynamics Specialist Conference and Exhibit, Keystone, CO, Aug 2006.

${ }^{34}$ Hull, D. G., Optimal Control Theory for Applications, Springer-Verlag, New York, 2003.

${ }^{35}$ Davis, P., Interpolation \& Approximation, Dover Publications, 1975.

${ }^{36}$ Davis, P., Rabinowitz, P., Methods of Numerical Integration, Academic Press, 1984.

${ }^{37}$ Williams, P. , “A Gauss-Lobatto Quadrature Method For Solving Optimal Control Problems,” Australia Mathematical Society, ANZIAM J.47, pp.C101-C115, July, 2006.

${ }^{38}$ Benson, D., A Gauss Pseudospectral Transcription for Optimal Control, Ph.D. Dissertation, Department of Aeronautics and Astronautics, Massachusetts Institute of Technology, November 2004.

${ }^{39}$ Gill, P.E., Murray, W., and Saunders, M.A., "SNOPT: An SQP Algorithm for Large Scale Constrained Optimization,” SIAM Journal on Optimization, Vol. 12, No. 4, 2002 
${ }^{40}$ Betts, John T., Practical Methods for Optimal Control Using Nonlinear Programming , SIAM. (2001)

${ }^{41}$ Huntington, G. T., Benson, D. A., and Rao, A. V., "Post-Optimality Evaluation and Analysis of a Formation Flying Problem via a Gauss Pseudospectral Method," Proceedings of the 2005 AAS/AIAA Astrodynamics Specialist Conference, AAS Paper 05-339, Lake Tahoe, California, August 7-11, 2005.

${ }^{42}$ Bryson, A., Ho, Y., Applied Optimal Control, Blaisdell Publishing Co. (1969) 


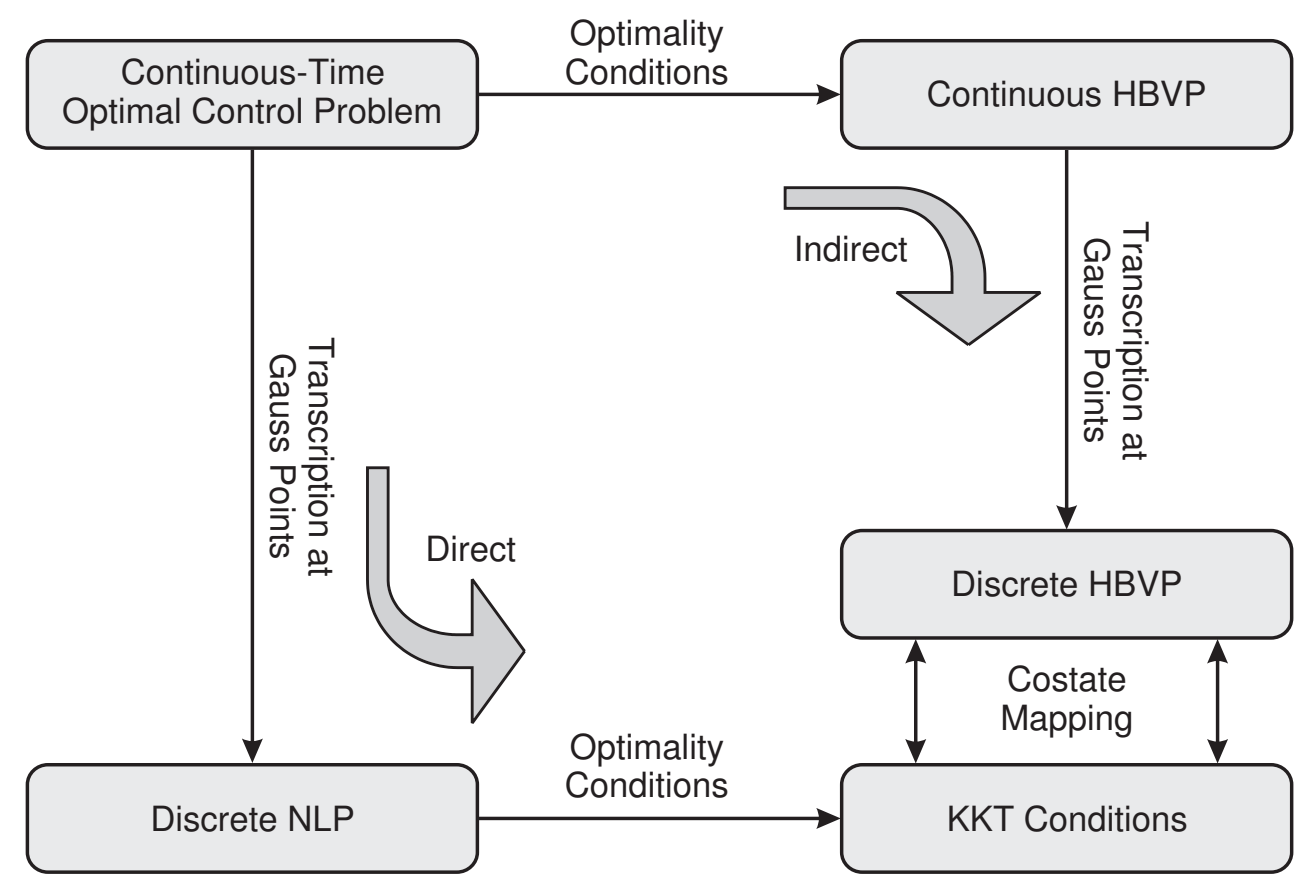

Figure 1: Direct vs Indirect Flow Diagram. 


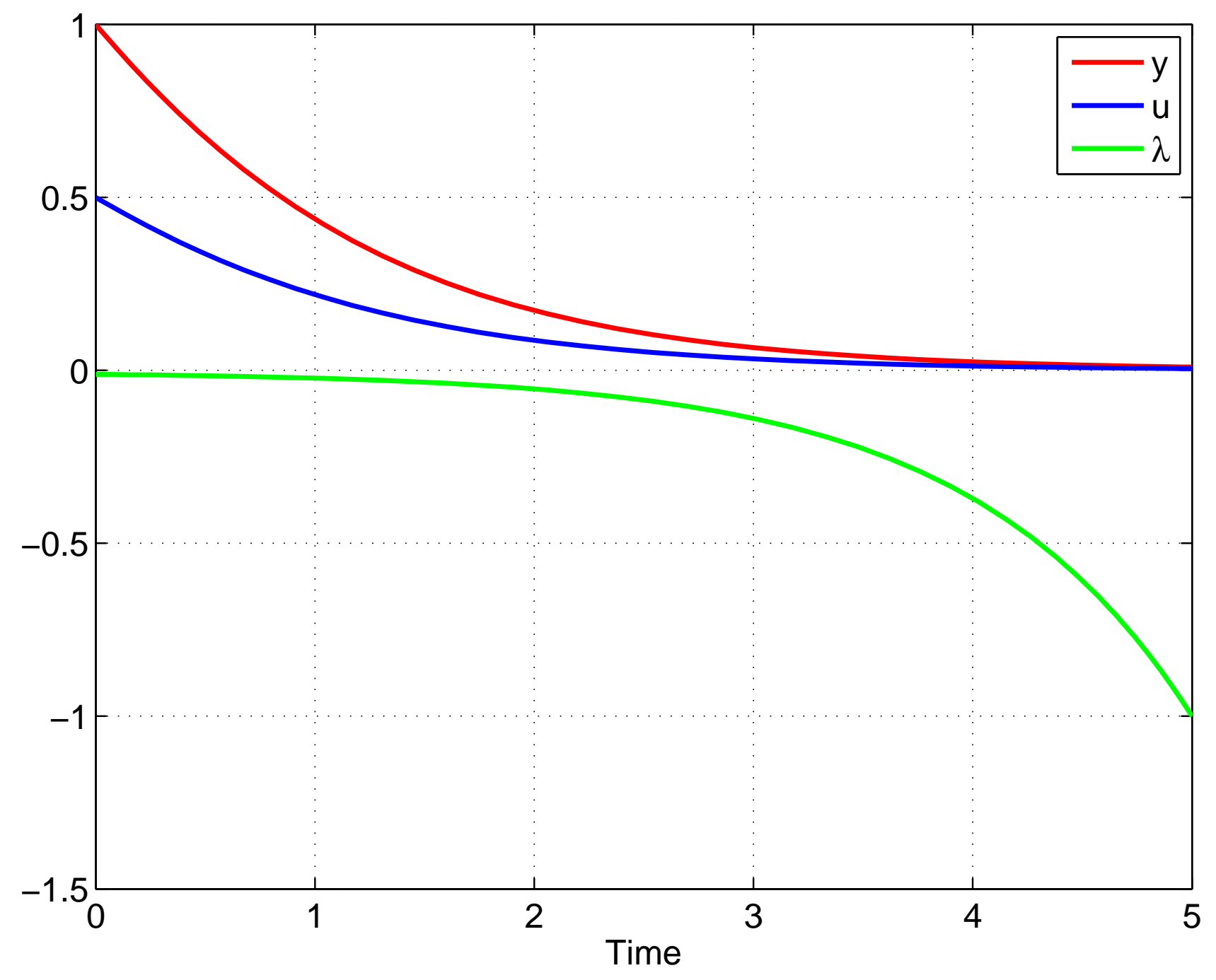

Figure 2: 1state exact. 


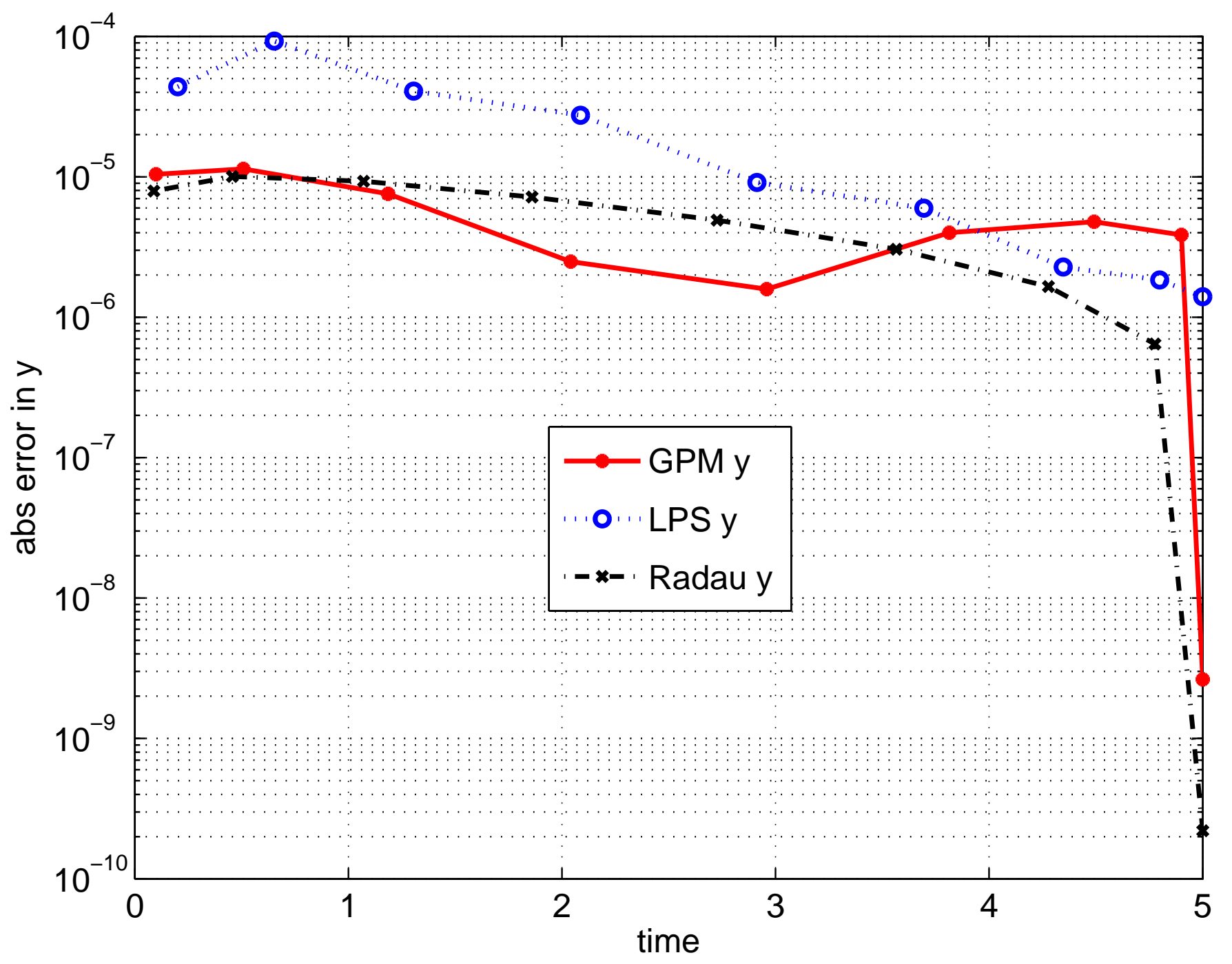

Figure 3: 1state absyerr. 


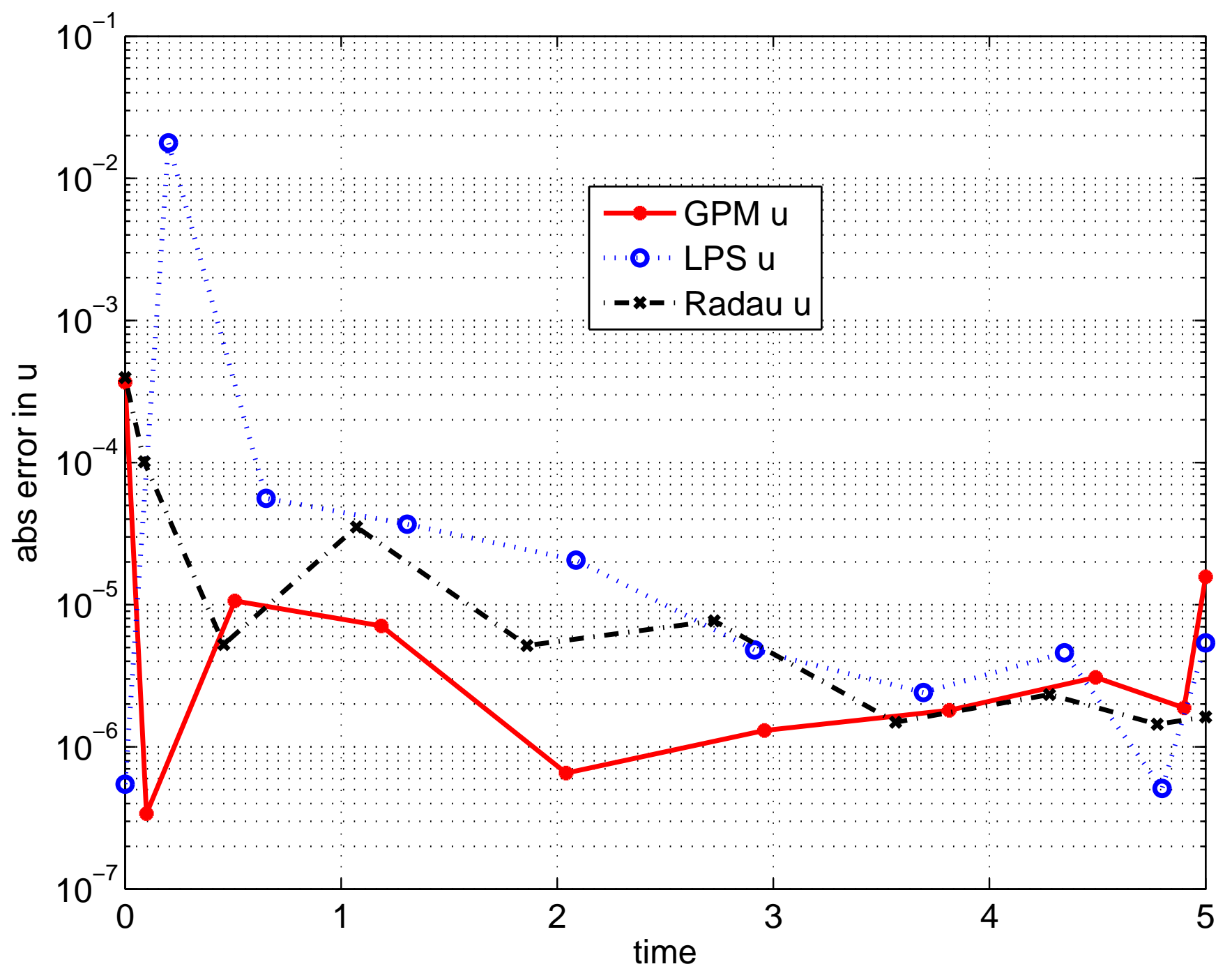

Figure 4: 1state absuerr. 


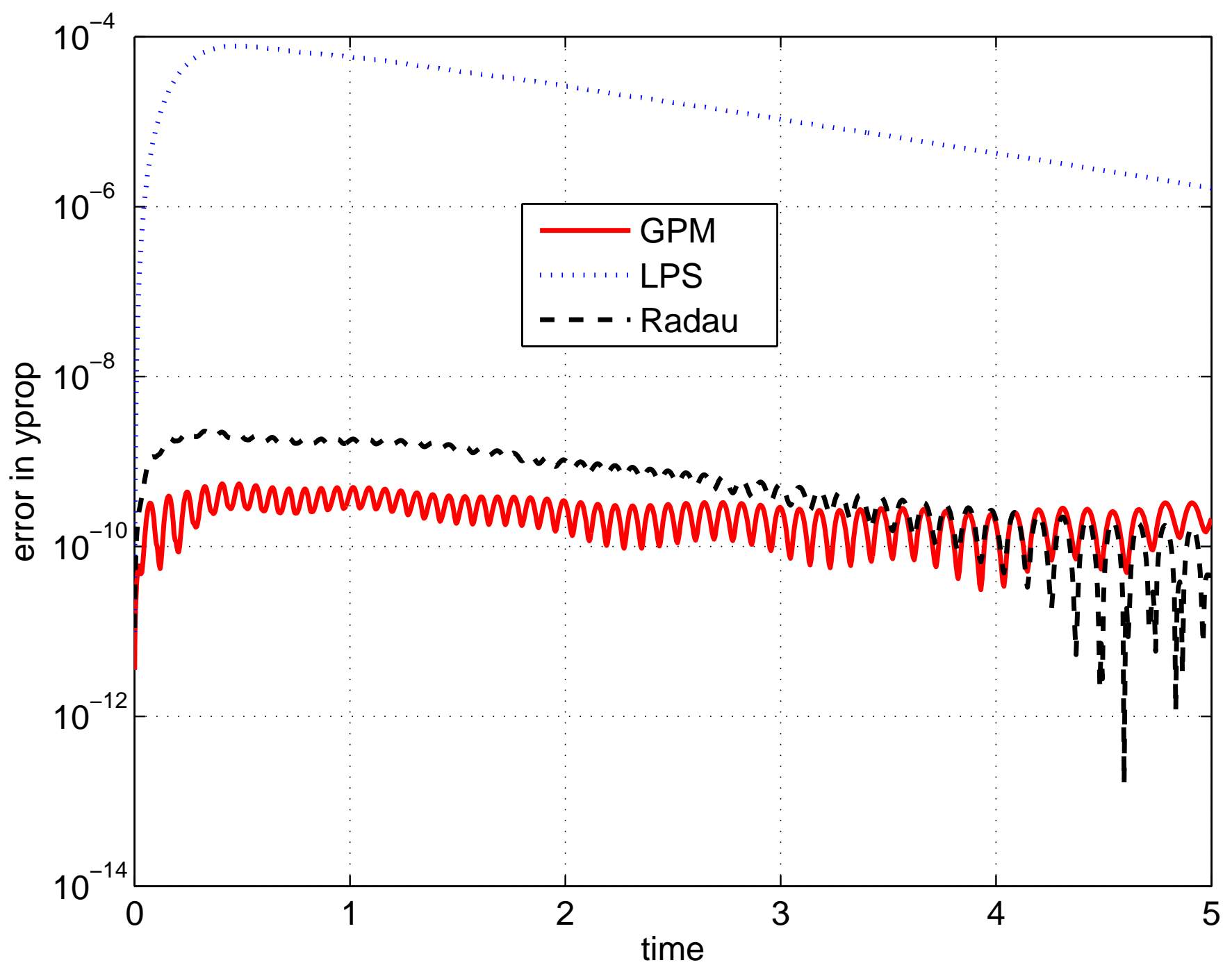

Figure 5: 1state yproperr. 


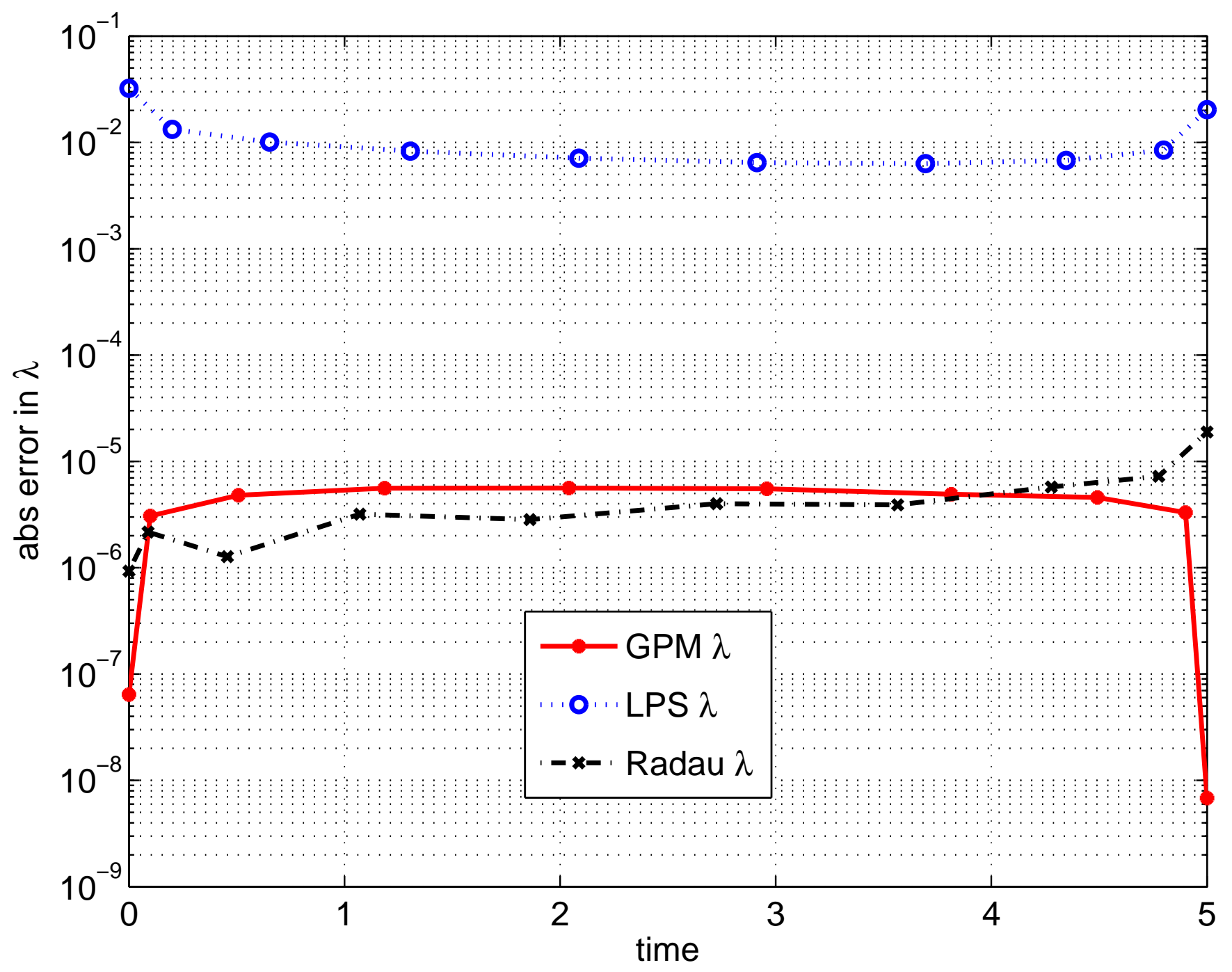

Figure 6: 1state abslamerr. 


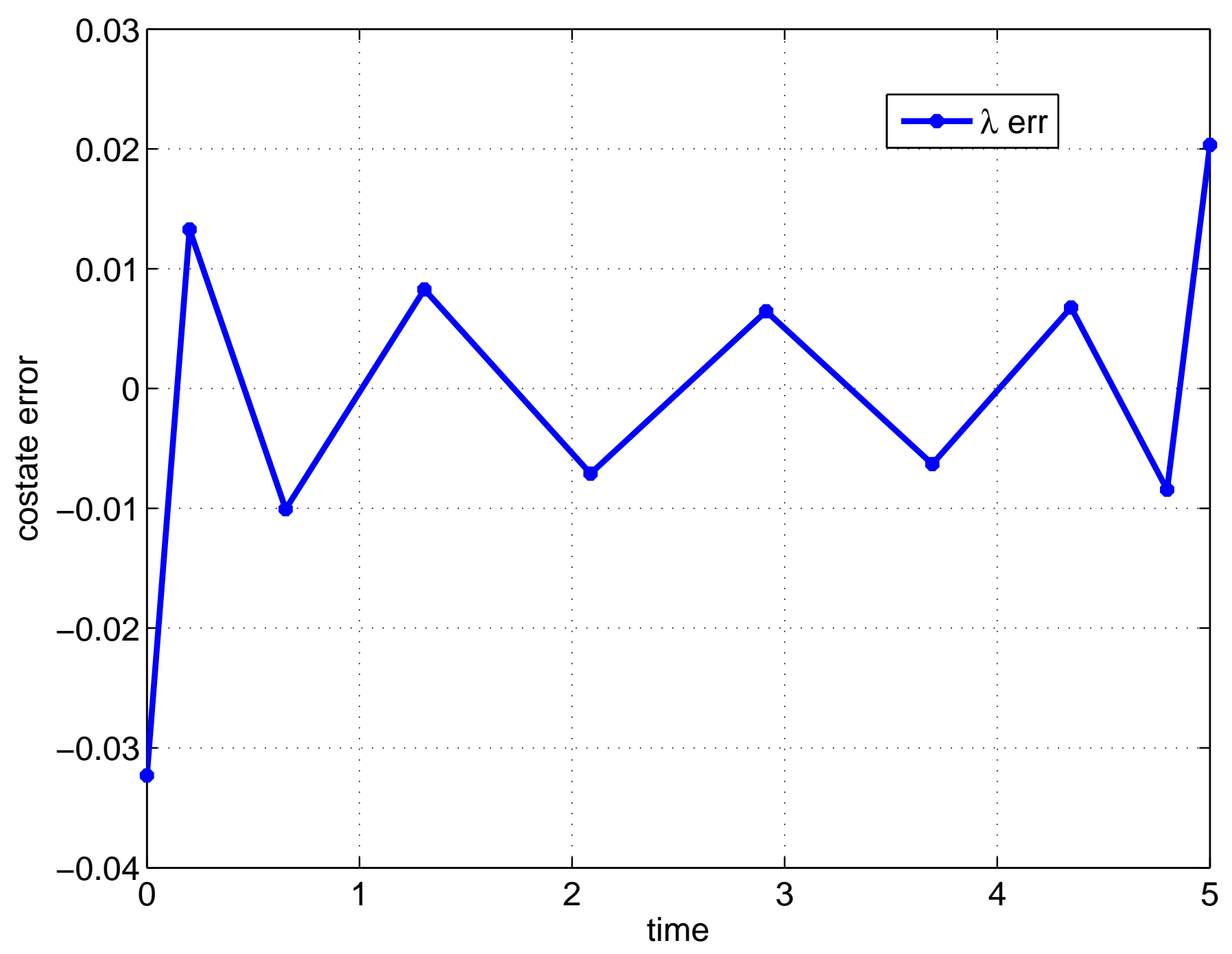

Figure 7: 1state lamerr. 


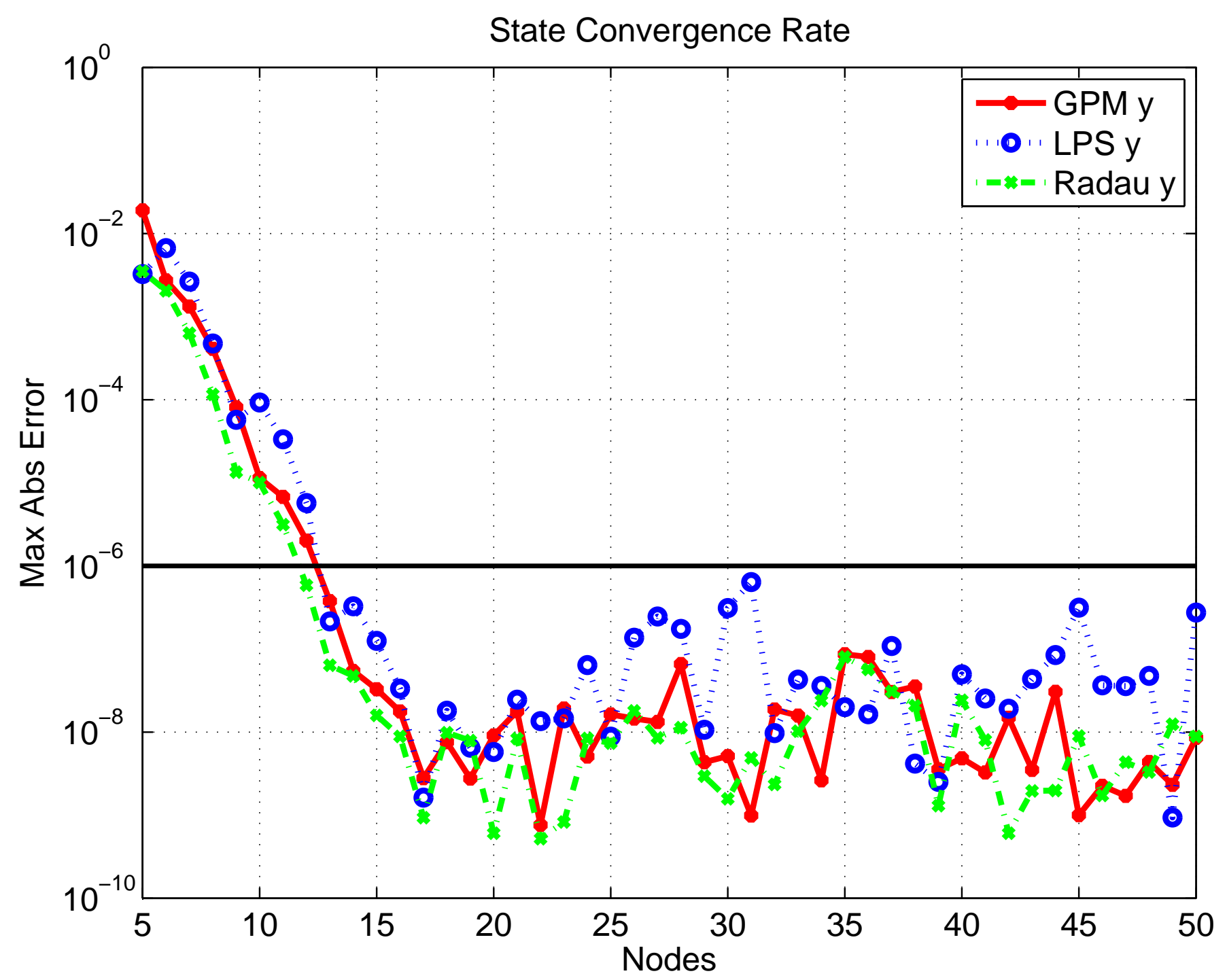

Figure 8: 1state convergence y. 


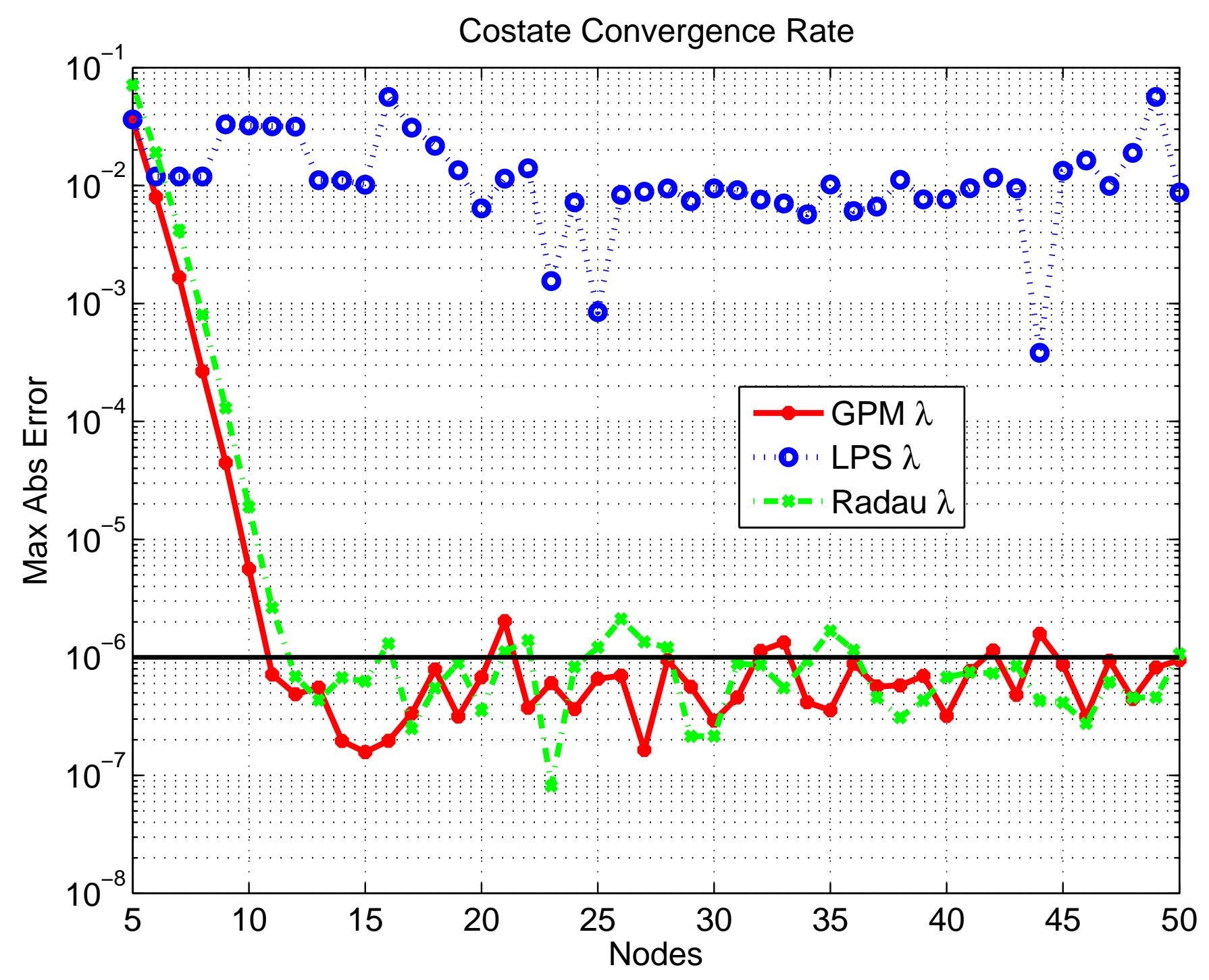

Figure 9: 1state convergence lam. 


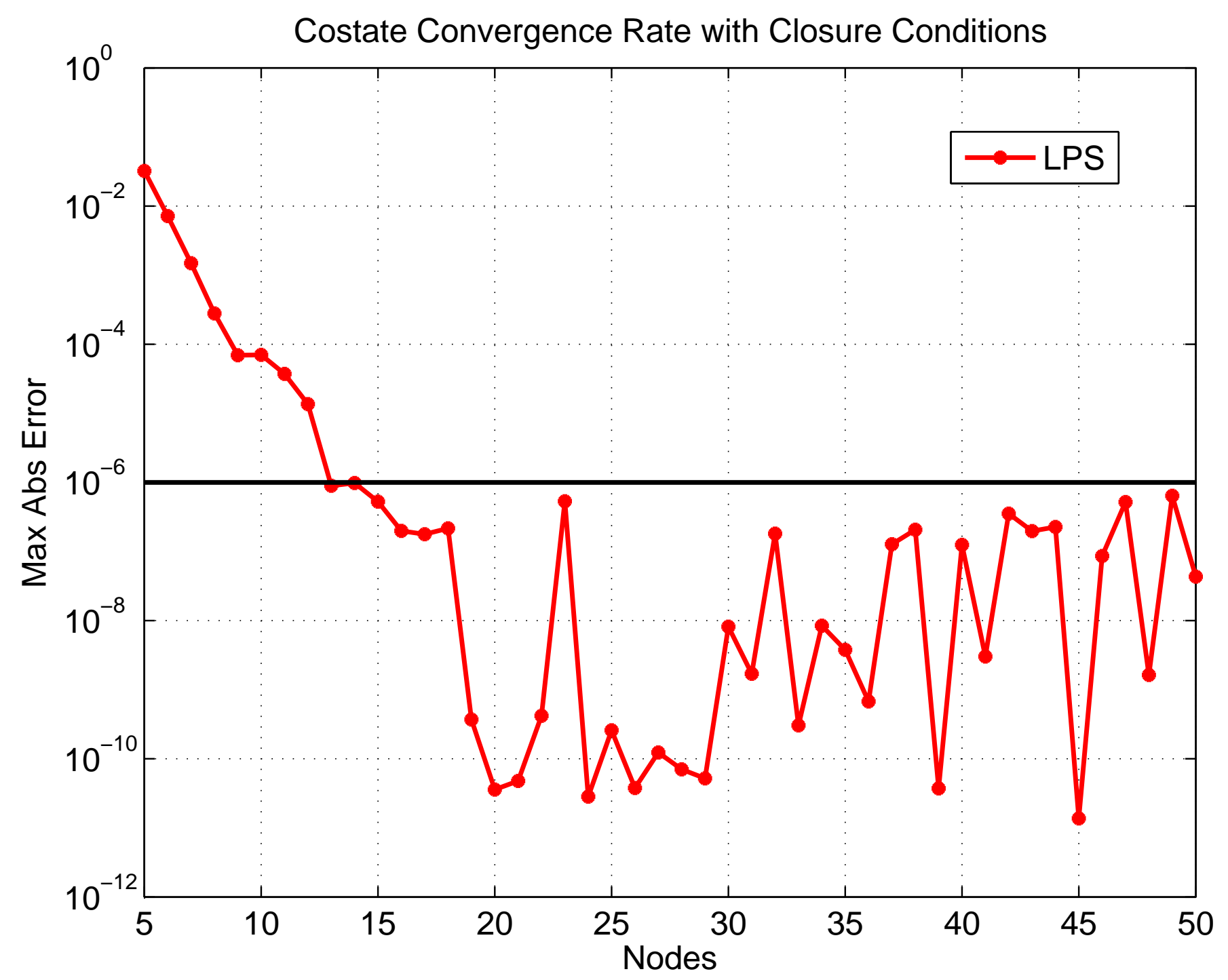

Figure 10: 1state CC lam. 


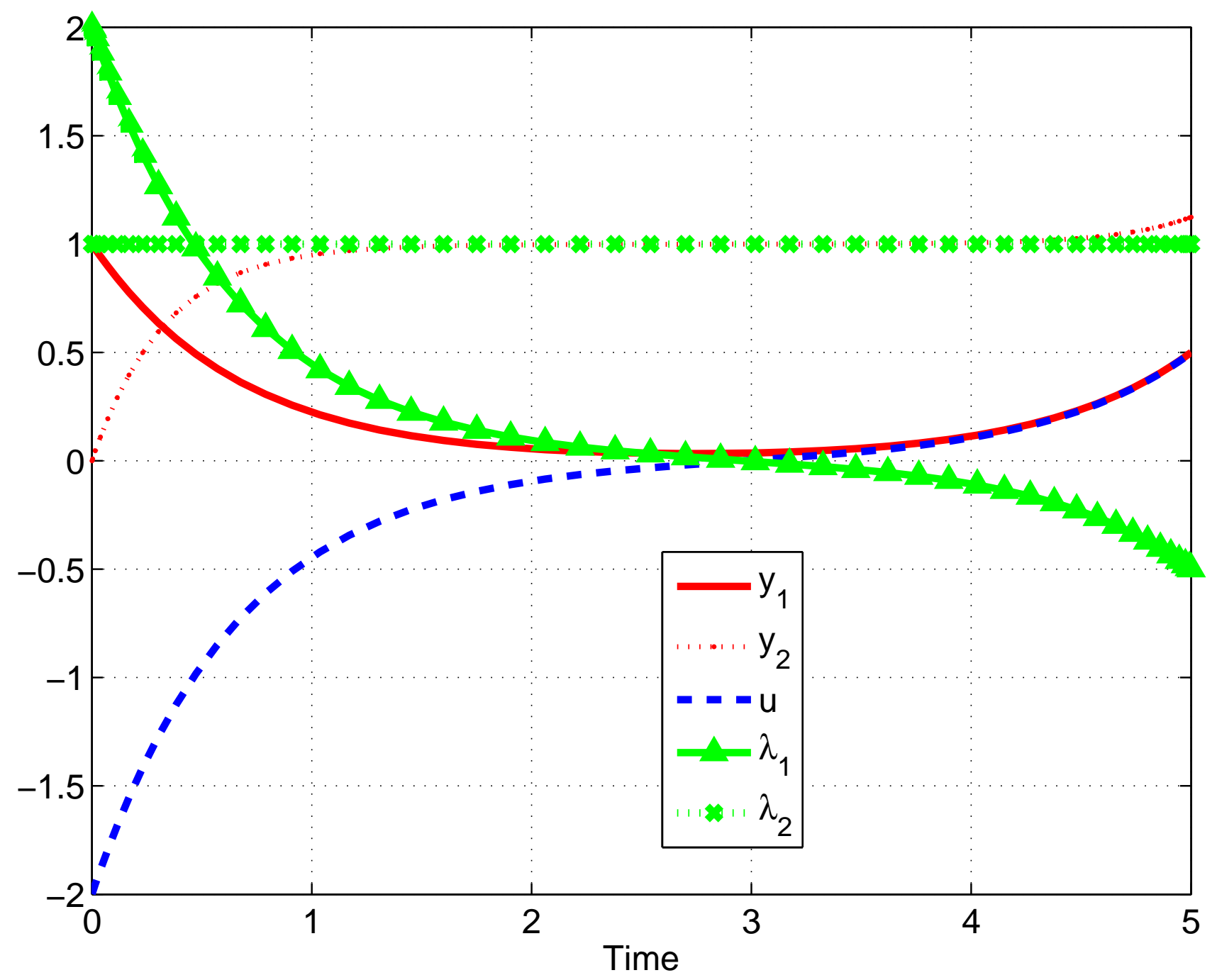

Figure 11: 2state exact. 


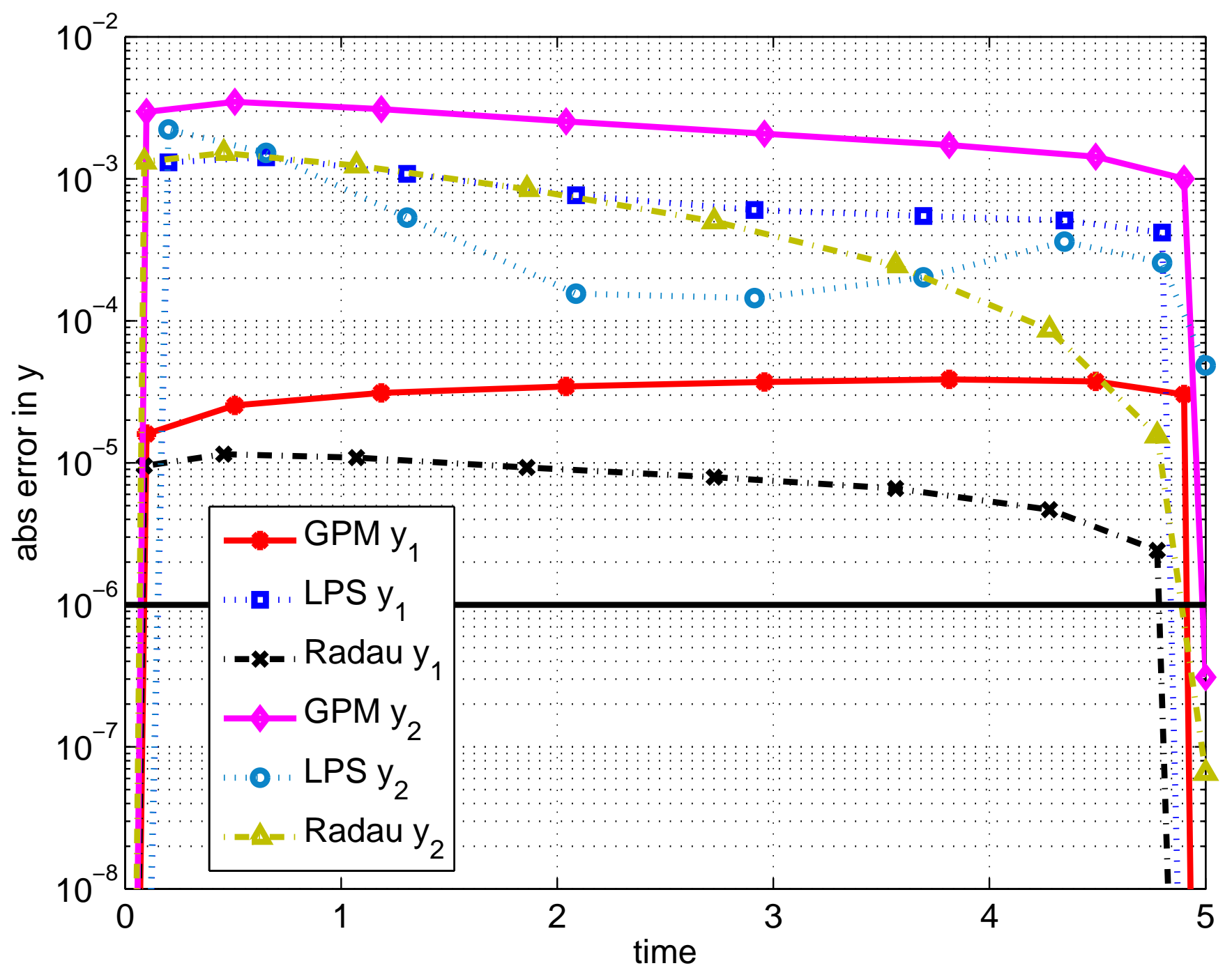

Figure 12: 2state absyerr. 


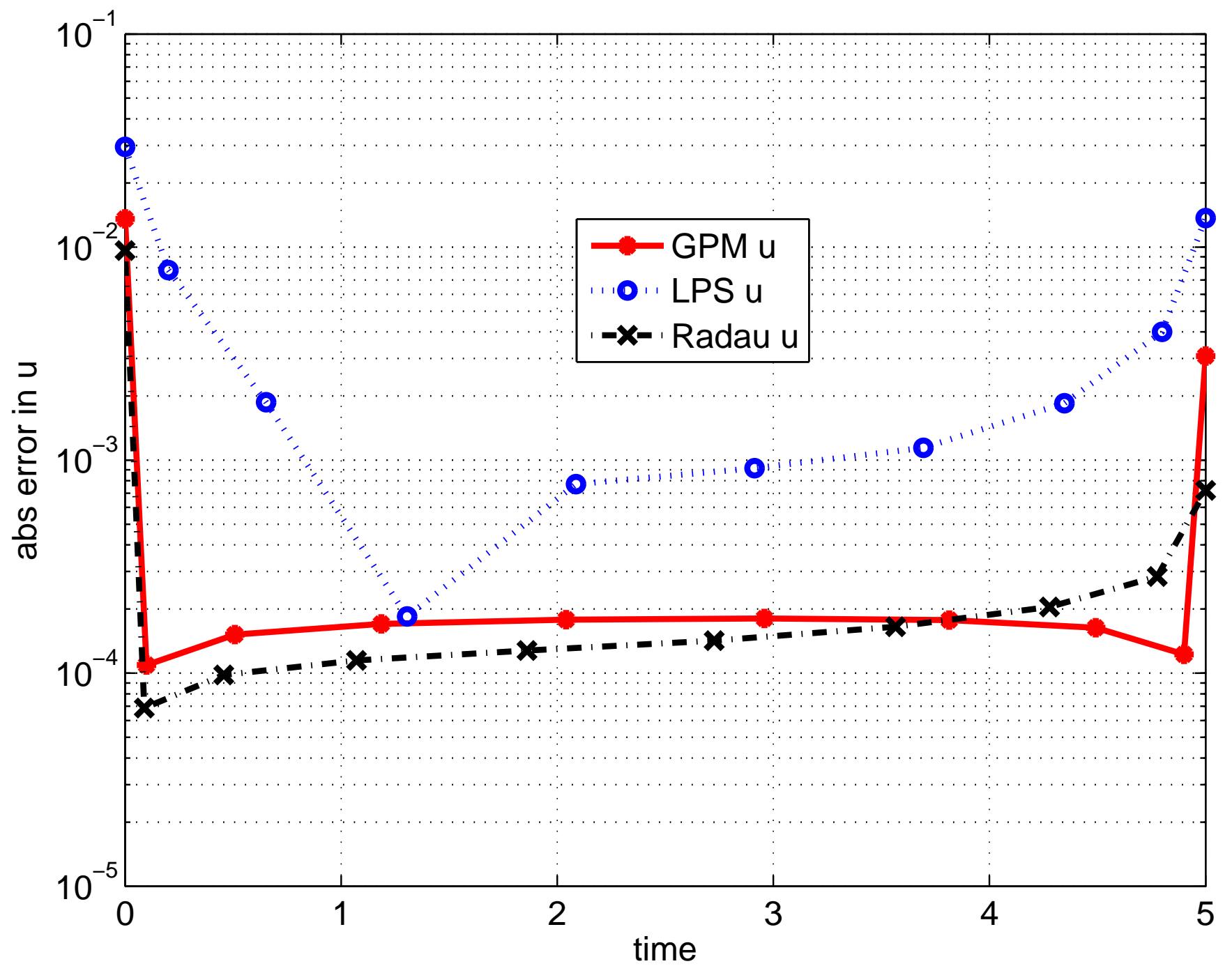

Figure 13: 2state absuerr. 


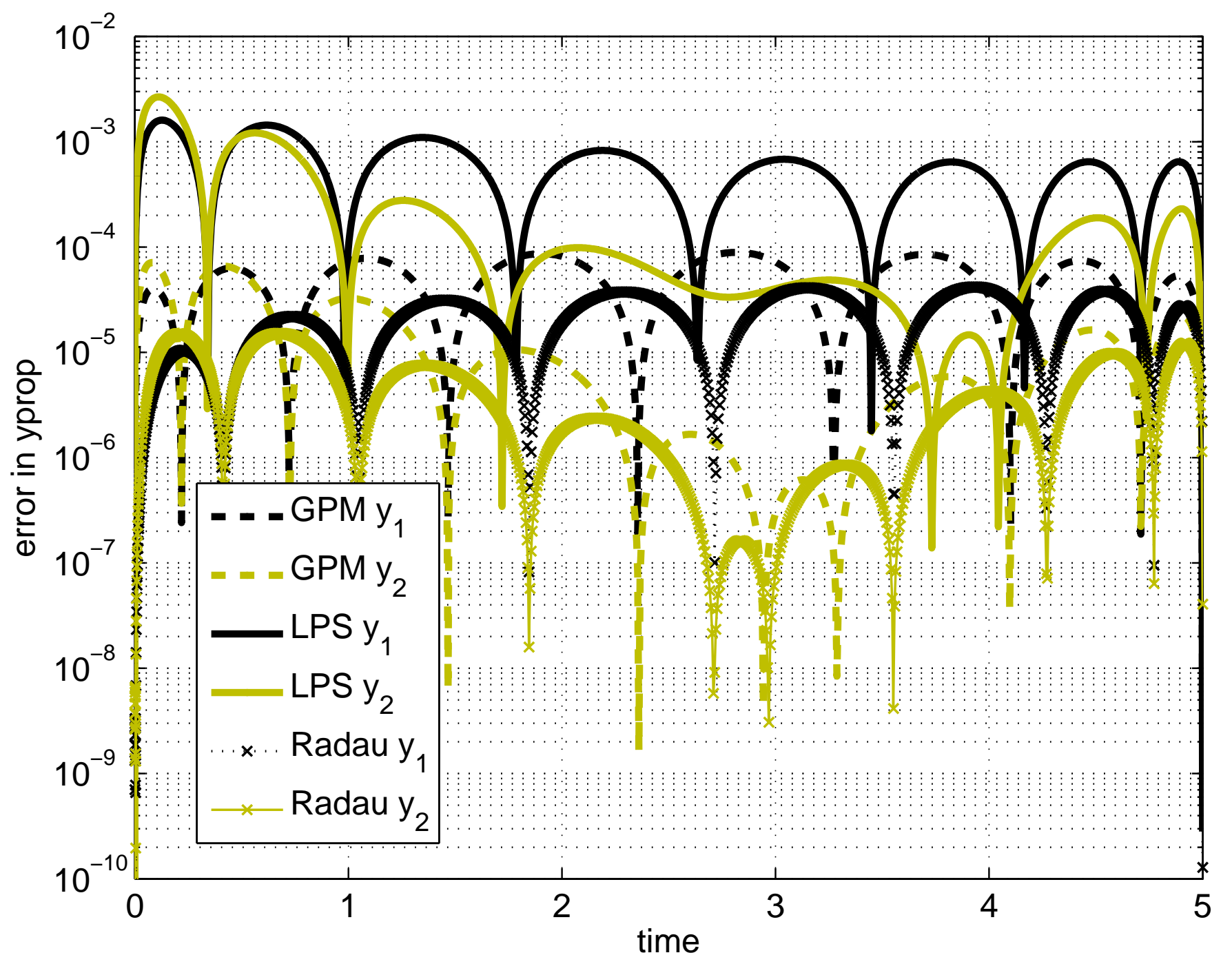

Figure 14: 2state yproperr. 


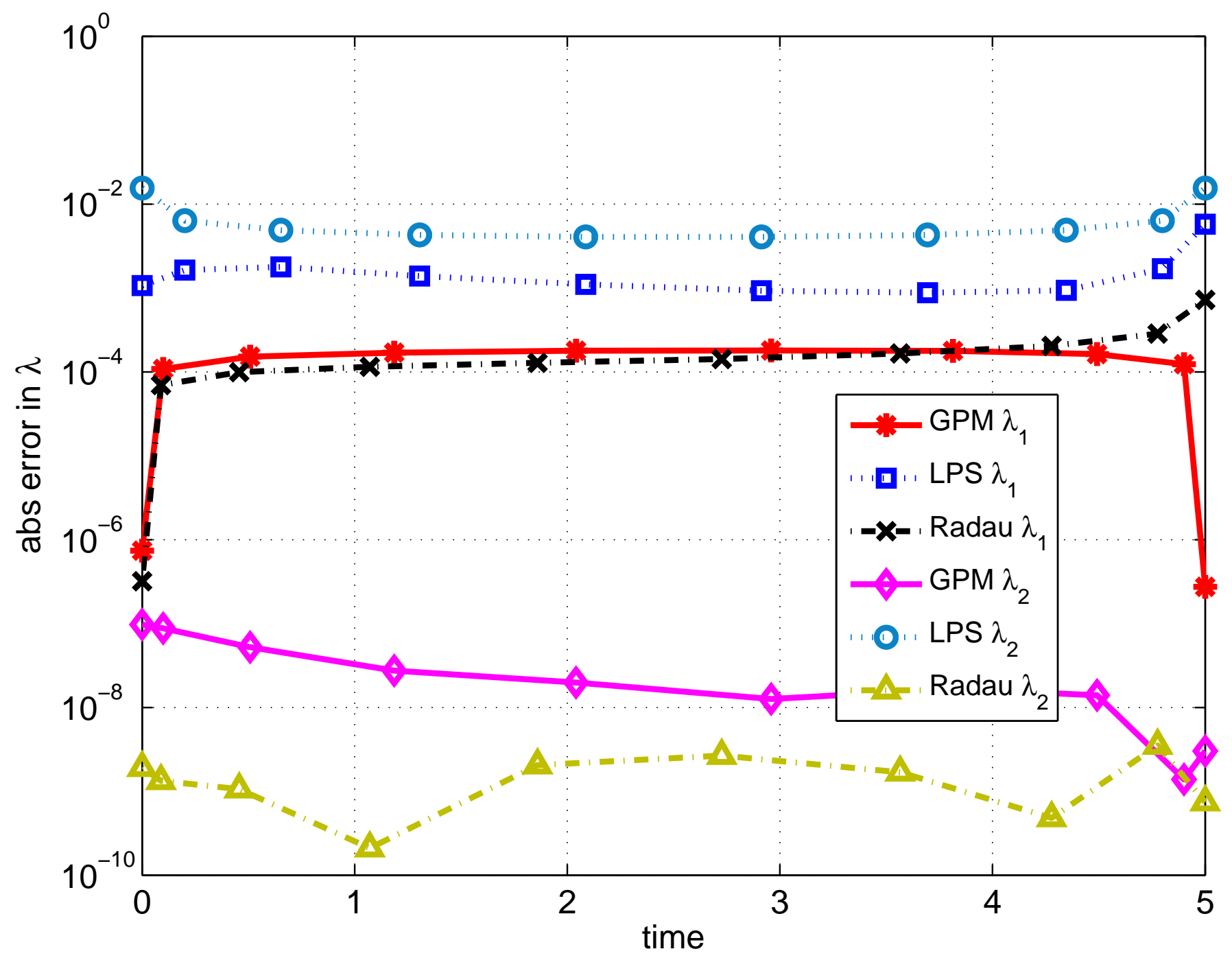

Figure 15: 2state abslamerr. 


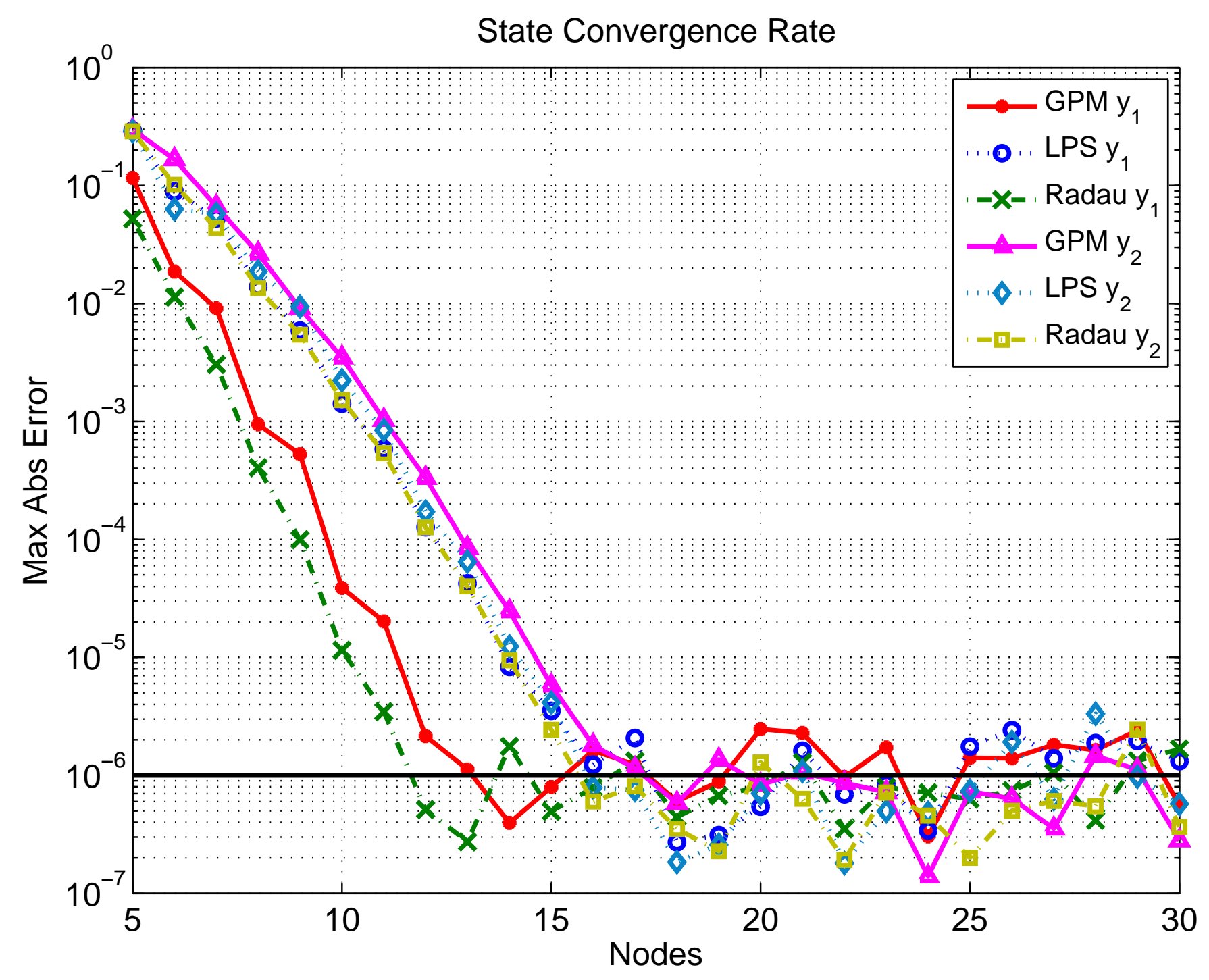

Figure 16: 2state convergence y. 


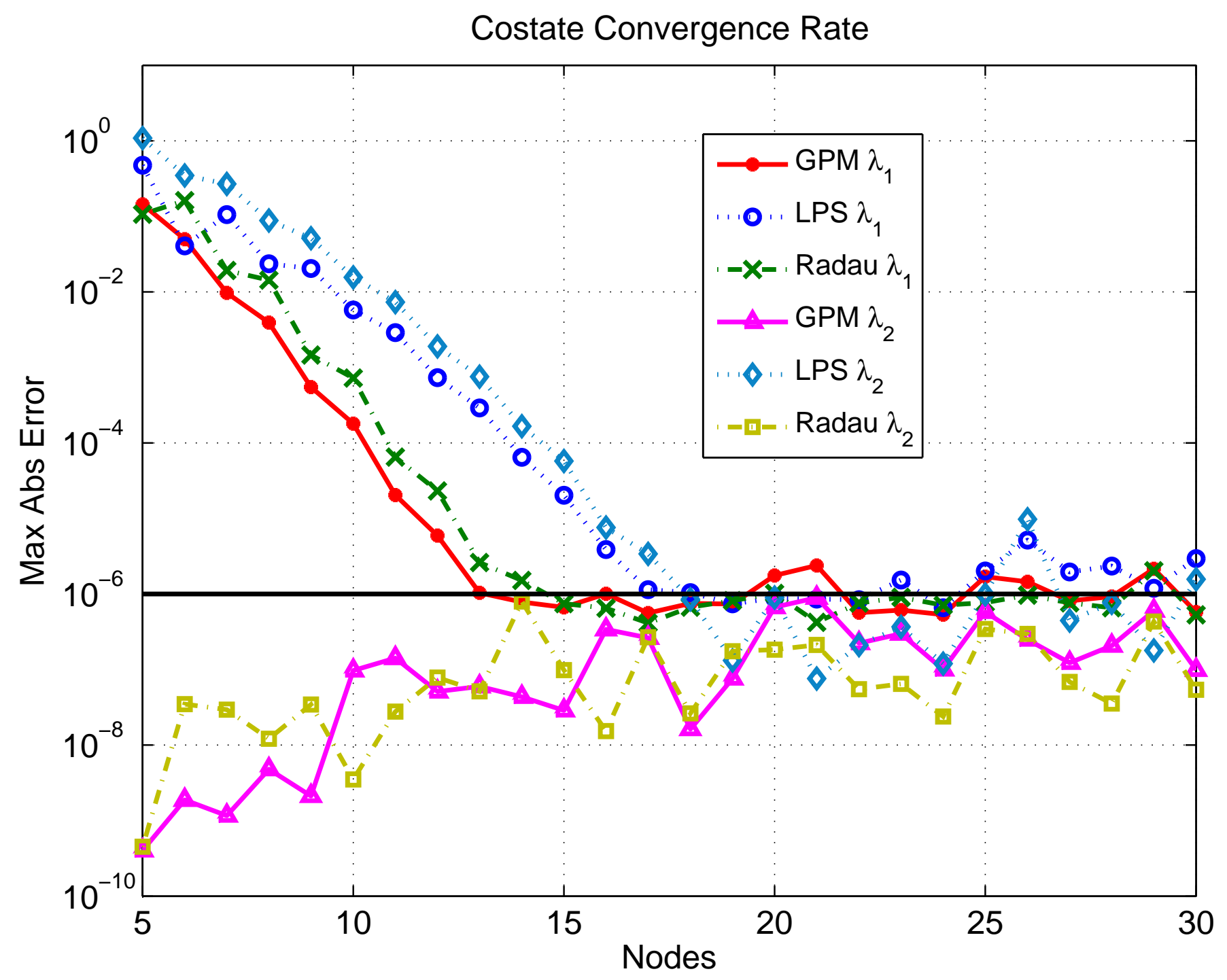

Figure 17: 2state convergence lam. 


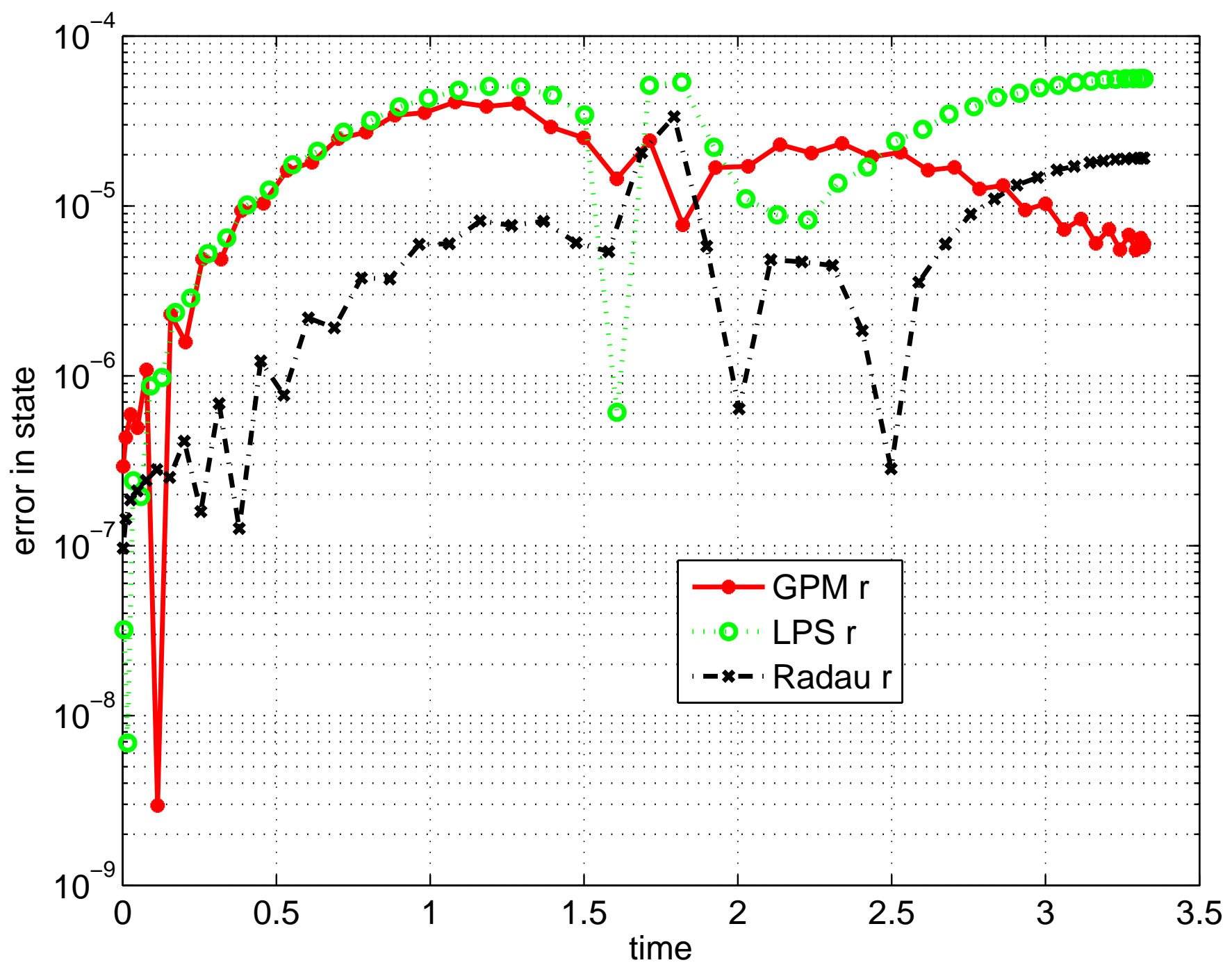

Figure 18: error in state $\mathrm{r}$. 


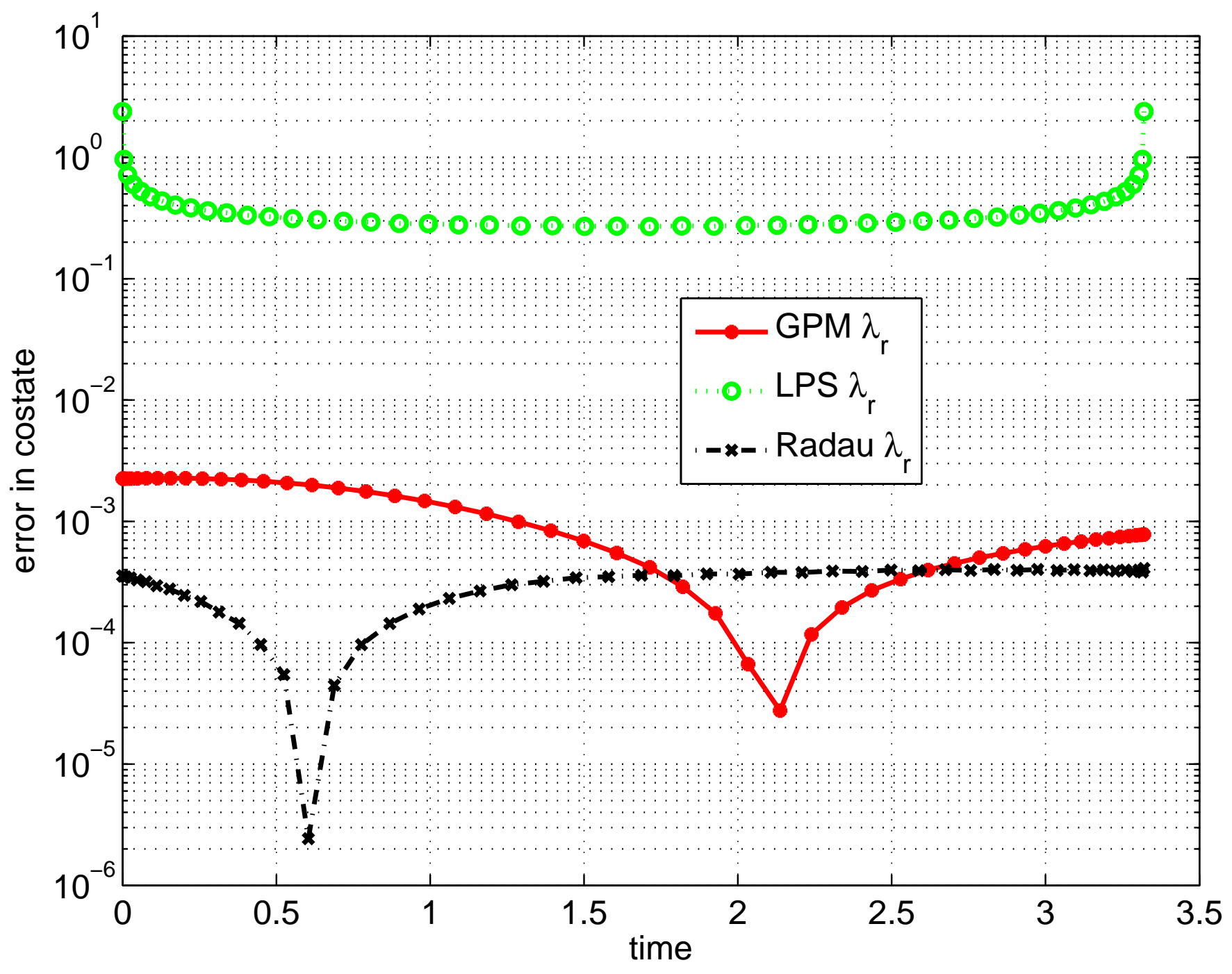

Figure 19: error in costate lamr . 


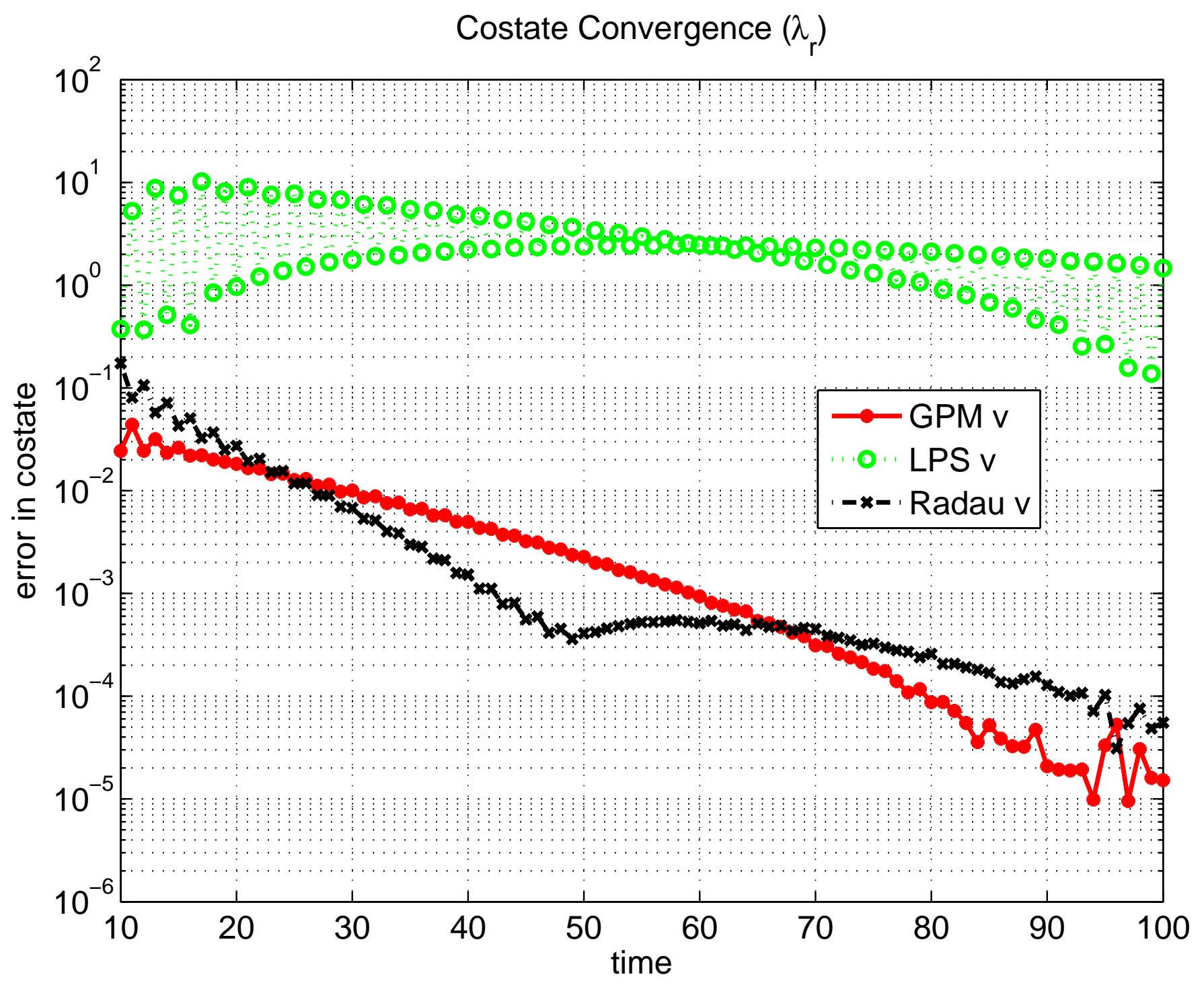

Figure 20: convergence of costate lamr . 
Table 1: Definitions of Lagrange interpolation polynomials used in this work

\begin{tabular}{|c|c|c|c|}
\hline \# of Basis Polynomials & Application & Symbol & Index \\
\hline $\mathrm{N}$ & $\operatorname{LPM}(\mathbf{x}, \mathbf{u}, \boldsymbol{\lambda})$ & $\mathcal{L}_{i}(\boldsymbol{\tau})$ & $i=1, \ldots, N$ \\
\hline $\mathrm{N}$ & $\operatorname{RPM}(\mathbf{x})$ & $\mathcal{L}_{i}(\boldsymbol{\tau})$ & $i=0, \ldots, N-1$ \\
\hline $\mathrm{N}-1$ & $\mathrm{RPM}(\mathbf{u}, \boldsymbol{\lambda})$ & $\overline{\mathcal{L}}_{i}(\boldsymbol{\tau})$ & $i=1, \ldots, N-1$ \\
\hline $\mathrm{N}-1$ & $\mathrm{GPM}(\mathbf{x}, \boldsymbol{\lambda})$ & $\overline{\mathcal{L}}_{i}(\boldsymbol{\tau})$ & $i=0, \ldots, N-2$ \\
\hline $\mathrm{N}-2$ & $\mathrm{GPM}(\mathbf{u})$ & $\tilde{\mathcal{L}}_{i}(\boldsymbol{\tau})$ & $i=1, \ldots, N-2$ \\
\hline
\end{tabular}


Table 2: CPU Times for each example and each method, in seconds

\begin{tabular}{|c||c|c|c|}
\hline & GPM & RPM & LPM \\
\hline \hline Ex 1 $(N=50)$ & 4.265 & 4.203 & 4.750 \\
\hline Ex 2 $(N=30)$ & 2.203 & 2.296 & 2.688 \\
\hline Ex 3 $(N=40)$ & 3.969 & 4.046 & 5.156 \\
\hline
\end{tabular}

